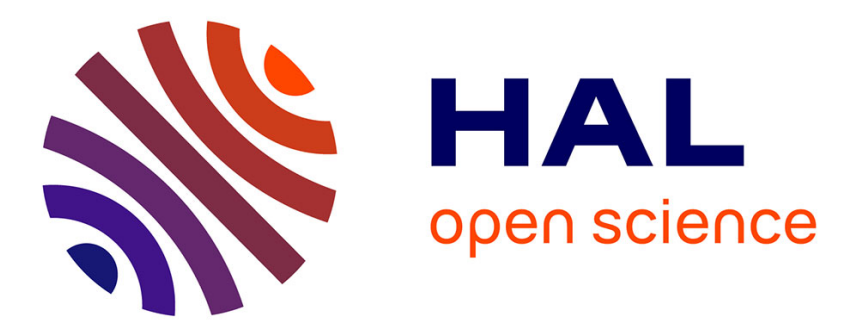

\title{
Some well-posedness and general stability results in Timoshenko systems with infinite memory and distributed time delay
}

Aissa Guesmia

\section{- To cite this version:}

Aissa Guesmia. Some well-posedness and general stability results in Timoshenko systems with infinite memory and distributed time delay. Journal of Mathematical Physics, 2014, 55 (8), 10.1063/1.4891489 . hal-01281853

\section{HAL Id: hal-01281853 \\ https://inria.hal.science/hal-01281853}

Submitted on 2 Mar 2016

HAL is a multi-disciplinary open access archive for the deposit and dissemination of scientific research documents, whether they are published or not. The documents may come from teaching and research institutions in France or abroad, or from public or private research centers.
L'archive ouverte pluridisciplinaire HAL, est destinée au dépôt et à la diffusion de documents scientifiques de niveau recherche, publiés ou non, émanant des établissements d'enseignement et de recherche français ou étrangers, des laboratoires publics ou privés. 


\section{AIP | Jounal of Mathematical Physics}

Some well-posedness and general stability results in Timoshenko systems with infinite memory and distributed time delay

Aissa Guesmia

Citation: Journal of Mathematical Physics 55, 081503 (2014); doi: 10.1063/1.4891489

View online: http://dx.doi.org/10.1063/1.4891489

View Table of Contents: http://scitation.aip.org/content/aip/journal/jmp/55/8?ver=pdfcov

Published by the AIP Publishing

\section{Articles you may be interested in}

Energy decay result in a Timoshenko-type system of thermoelasticity of type III with distributive delay J. Math. Phys. 54, 101503 (2013); 10.1063/1.4826102

Traveling wave governs the stability of spatial pattern in a model of allelopathic competition interactions

Chaos 22, 043136 (2012); 10.1063/1.4770064

Global well-posedness of a dissipative system arising in electrohydrodynamics in negative-order Besov spaces

J. Math. Phys. 51, 093101 (2010); 10.1063/1.3484184

Inverse synchronizations in coupled time-delay systems with inhibitory coupling

Chaos 19, 023107 (2009); 10.1063/1.3125721

Stability analysis of neural networks with interval time-varying delays

Chaos 17, 033120 (2007); 10.1063/1.2771082

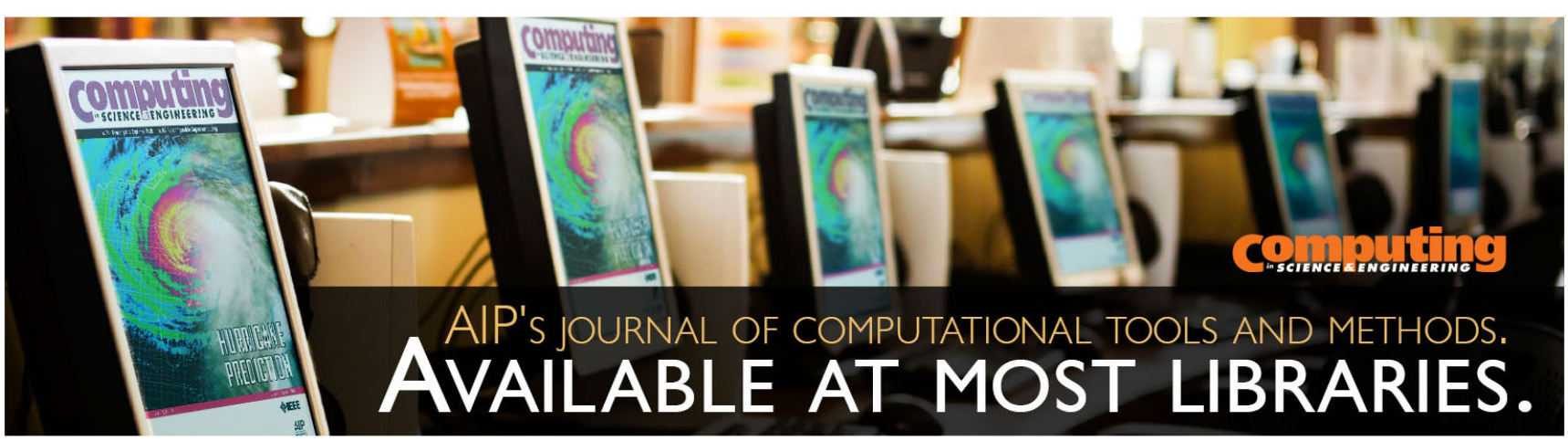




\title{
Some well-posedness and general stability results in Timoshenko systems with infinite memory and distributed time delay
}

\author{
Aissa Guesmia ${ }^{a)}$ \\ Elie Cartan Institute of Lorraine, UMR 7502, University of Lorraine, Bat. A, Ile du Saulcy, \\ 57045 Metz Cedex 01, France
}

(Received 6 March 2014; accepted 16 July 2014; published online 11 August 2014)

\begin{abstract}
In this paper, we consider a Timoshenko system in one-dimensional bounded domain with infinite memory and distributed time delay both acting on the equation of the rotation angle. Without any restriction on the speeds of wave propagation and under appropriate assumptions on the infinite memory and distributed time delay convolution kernels, we prove, first, the well-posedness and, second, the stability of the system, where we present some decay estimates depending on the equalspeed propagation case and the opposite one. The obtained decay rates depend on the growths of the memory and delay kernels at infinity. In the nonequal-speed case, the decay rate depends also on the regularity of initial data. Our stability results show that the only dissipation resulting from the infinite memory guarantees the asymptotic stability of the system regardless to the speeds of wave propagation and in spite of the presence of a distributed time delay. Applications of our approach to specific coupled Timoshenko-heat and Timoshenko-wave systems as well as the discrete time delay case are also presented. (C) 2014 AIP Publishing LLC. [http://dx.doi.org/10.1063/1.4891489]
\end{abstract}

\section{INTRODUCTION}

Let $g: \mathbb{R}_{+} \rightarrow \mathbb{R}_{+}$and $f: \mathbb{R}_{+} \rightarrow \mathbb{R}$ be given functions. We consider the following Timoshenko system:

$$
\left\{\begin{array}{l}
\rho_{1} \varphi_{t t}(x, t)-k_{1}\left(\varphi_{x}(x, t)+\psi(x, t)\right)_{x}=0, \\
\rho_{2} \psi_{t t}(x, t)-k_{2} \psi_{x x}(x, t)+k_{1}\left(\varphi_{x}(x, t)+\psi(x, t)\right) \\
\quad+\int_{0}^{+\infty} g(s) \psi_{x x}(x, t-s) d s+\int_{0}^{+\infty} f(s) \psi_{t}(x, t-s) d s=0, \\
\varphi(0, t)=\psi(0, t)=\varphi(L, t)=\psi(L, t)=0, \\
\varphi(x, 0)=\varphi_{0}(x), \quad \varphi_{t}(x, 0)=\varphi_{1}(x), \\
\psi(x,-t)=\psi_{0}(x, t), \quad \psi_{t}(x,-t)=\psi_{1}(x, t),
\end{array}\right.
$$

where $(x, t) \in] 0, L\left[\times \mathbb{R}_{+},\left(\varphi_{0}, \psi_{0}, \varphi_{1}, \psi_{1}\right)\right.$ are given initial data belonging to a suitable space, $(\varphi$, $\psi$ ) is the state (unknown) of (1.1), $L, \rho_{1}, \rho_{2}, k_{1}$, and $k_{2}$ are positive constants. A subscript $y$ denotes the derivative with respect to $y$. We also use the prime notation to denote the derivative when the function has only one variable. The infinite integrals depending on $g$ and $f$ represent, respectively, the infinite memory and the distributed time delay terms. This type of systems has been introduced in Ref. 44. It describes the transverse vibration of a thick beam of length $L$, where $\varphi$ is the transverse displacement of the beam, $-\psi$ is the rotation angle of the filament of the beam, and $\rho_{1}, \rho_{2}, k_{1}$, and $k_{2}$ account for some physical properties of the beam (see, for example, Refs. 23 and 24).

\footnotetext{
a) Also at Department of Mathematics and Statistics, College of Sciences, King Fahd University of Petroleum and Minerals, P. O. Box. 5005, Dhahran 31261, Saudi Arabia. Electronic addresses: guesmia@univ-metz.fr and guesmia@kfupm.edu.sa
} 
Our objective here is to prove the well-posedness and investigate the asymptotic behavior as time goes to infinity of solutions of (1.1) under appropriate assumptions on the convolution kernels $g$ and $f$.

The questions related to well-posedness and stability/instability of evolution equations with delay and/or memory have attracted considerable attention in recent years and many researchers have shown that the memory plays the role of a damper, whereas the time delay can destabilize a system that was asymptotically stable in the absence of time delay. The main problem concerning the stability in the presence of memory is determining the largest class of kernels $g$ which guarantee the stability and the best relation between the decay rate of $g$ and the asymptotic behavior of solutions of the considered system. Because a small delay time can be a source of instability (see, for example, Ref. 28), to stabilize a hyperbolic system involving input delay terms, additional control terms (like memory or frictional damping) will be necessary. Let us recall some works related to the subject of the present paper.

In the absence of time delay term (i.e., $f \equiv 0$ ), a large amount of the literature is available on Timoshenko-type systems ${ }^{44}$ with (finite or infinite) memory or frictional damping, addressing the issues of the existence, uniqueness, smoothness, and asymptotic behavior in time; see, for example, Refs. 2, 4, 5, 8, 9, 16-19, 25, and 26, and the references cited therein. In these papers, it was shown that the dissipation given by the memory term is strong enough to stabilize the system, and various decay estimates (exponential, polynomial, or others) have been obtained depending on the regularity of the initial data, the growth of $g$ at infinity and the relation

$$
\frac{k_{1}}{\rho_{1}}=\frac{k_{2}}{\rho_{2}}
$$

and the opposite one. The equality (1.2) means that the first two equations of (1.1) have the same speeds of wave propagation $\sqrt{\frac{k_{1}}{\rho_{1}}}$ and $\sqrt{\frac{k_{2}}{\rho_{2}}}$, respectively. Similar stability results are known in the literature for other hyperbolic evolution equations with memory; see, for example, Ref. 14 and the references cited therein. The idea of proof consists in considering some integral and/or differenial inequalities involving $g$ and/or some of its derivatives as a characterization of the growth of $g$ at infinity from which the decay rate of the solution is deduced.

When the second equation of (1.1) is replaced by

$$
\begin{gathered}
\rho_{2} \psi_{t t}(x, t)-k_{2} \psi_{x x}(x, t)+k_{1}\left(\varphi_{x}(x, t)+\psi(x, t)\right) \\
+\int_{0}^{t} g(s) \psi_{x x}(x, t-s) d s+\mu_{1} \psi_{t}(x, t)+\mu_{2} \psi_{t}(x, t-\tau)=0,
\end{gathered}
$$

where $\mu_{1}, \mu_{2}$, and $\tau$ are fixed non-negative constants, the stability of Timoshenko system was proved in Ref. 35 under the assumption $0 \leq \mu_{2} \leq \mu_{1}$. The decay rate of solution obtained in Ref. 35 depends on the one of $g$. This result shows that the dissipation resulting from both finite memory $\int_{0}^{t} g(s) \psi_{x x}(x, t-s) d s$ and frictional damping $\mu_{1} \psi_{t}(x, t)$ is strong enough to stabilize Timoshenko system in presence of a constant discrete time delay $\mu_{2} \psi_{t}(x, t-\tau)$ provided that the coefficient of the delay is smaller or equal than the one of the damping. Similar stability results for various hyperbolic evolution equations with frictional damping and time delay exist in the literature, in this regard, we refer the reader to Refs. 3, 6, 7,21,27-31, and 32.

As far as we know, the problem of stability of Timoshenko system with infinite memory and distributed time delay considered in this paper has never been treated in the literature. The stability of the following abstract hyperbolic equation with infinite memory and discrete or distributed time delay:

$$
u_{t t}(t)+A u(t)-\int_{0}^{+\infty} g(s) A u(t-s) d s+\mu u_{t}(t-\tau) d s=0
$$


and

$$
u_{t t}(t)+A u(t)-\int_{0}^{+\infty} g(s) B u(t-s) d s+\int_{0}^{+\infty} f(s) u_{t}(t-s) d s=0
$$

was studied in, respectively, Refs. 15 and 20, and several decay estimates were proved depending on the growth of $g$ and $f$ at infinity, and the connection between the operators $A$ and $B$. But (1.4)(1.5) do not include (1.1), since the operators $A$ and $B$ are supposed to be definite positive in Refs. 15 and 20.

Unlike the discrete time delay models, which ignore the inherent memory effects, the distributed time delay considered in this paper do take into account the whole (infinite) past history of the solution. More precisely, we are in presence of an indefinite frictional damping which depends on all previous states (the past information is stored and used later). This is what makes the present case more realistic. In fact, the discrete case will be a special case which corresponds to the Dirac delta distribution kernel (at some time $\tau$ ).

According to the known results cited above, one main question naturally arises: is it possible for the memory term, which plays solely the role of dissipation in (1.1), to play the same role as a robust controller against the delay and stabilize (1.1), and is it possible to get the decay rate of solutions explicitly in term of, in particular, the connection between the delay and the memory kernels? As far as we know, this situation has never been considered before in the literature. In this paper, we shall prove, regardless of the speeds of wave propagation and under some appropriate assumptions on $g$ and $f$, that (1.1) is well-posed in an appropriate underlying space, and that the only dissipation generated by the infinite memory guarantees the asymptotic stability of (1.1) in spite of the presence of a distributed time delay. Moreover, the decay rate of solutions is explicitly found in terms of the growths of $g$ and $f$ at infinity. When (1.2) does not hold, the decay rate depends also on the regularity of initial data and, so, it can be improved by choosing initial data regular enough. The proof is based on the semigroup theory for the well-posedness, and the energy method for the stability. We introduce new functionals to get crucial estimates on the distributed time delay and the infinite memory, and overcome subsequently the difficulties generated by the nondissipativeness character of our system (1.1). Moreover, we will appeal to some ideas and arguments in Refs. 20 and 36-43. These ideas will, in particular, allow us to deal with some arbitrary decaying kernels without assuming explicit conditions on their derivatives. The approach presented in this paper can be applied to the case of finite memory and/or discrete time delay as well as other Timoshenko-type systems.

The plan of the paper is as follows. In Sec. II, we present our assumptions on $g$ and $f$, and state and prove the well-posedness of (1.1). Section III is devoted to the statement and proof of the asymptotic stability results of (1.1) under some additional assumptions on $g$ and $f$. Section IV will be devoted to some applications to the coupled Timoshenko-heat and Timoshenko-wave systems as well as to the discrete time delay case. Finally, in Sec. V, we discuss some general comments and issues.

\section{WELL-POSEDNESS}

In this section, we state our assumptions on $g$ and $f$, and prove the global existence, uniqueness, and smoothness of the solution of (1.1). We assume that

(A1) The function $g$ is of class $C^{1}\left(\mathbb{R}_{+}, \mathbb{R}_{+}\right)$, nonincreasing and satisfies

$$
g_{0}:=\int_{0}^{+\infty} g(s) d s<k_{2}
$$

Moreover, for some positive constant $\theta_{0}$,

$$
-g^{\prime}(s) \leq \theta_{0} g(s), \quad \forall s \in \mathbb{R}_{+}
$$


(A2) The function $f$ is of class $C^{1}\left(\mathbb{R}_{+}, \mathbb{R}\right)$ and satisfies, for some positive constant $\alpha$,

$$
|f(s)| \leq \alpha g(s) \quad \text { and } \quad\left|f^{\prime}(s)\right| \leq \alpha g(s), \quad \forall s \in \mathbb{R}_{+} .
$$

Following a method devised in Ref. 5, we consider a new auxiliary variable $\eta$ to treat the infinite memory and distributed time delay terms, and formulate the system (1.1) in the following abstract linear first-order system:

$$
\left\{\begin{array}{l}
\mathscr{U}_{t}(t)=(\mathscr{A}+\mathscr{B}) \mathscr{U}(t), \\
\mathscr{U}(0)=\mathscr{U}_{0},
\end{array}\right.
$$

where

$$
\begin{gathered}
\left\{\begin{array}{l}
\mathscr{U}=\left(\varphi, \psi, \varphi_{t}, \psi_{t}, \eta\right)^{T}, \\
\mathscr{U}_{0}=\left(\varphi_{0}, \psi_{0}(\cdot, 0), \varphi_{1}, \psi_{1}(\cdot, 0), \eta_{0}\right)^{T} \in \mathscr{H},
\end{array}\right. \\
\mathscr{H}=\left(H_{0}^{1}(] 0, L[)\right)^{2} \times\left(L^{2}(] 0, L[)\right)^{2} \times L_{g}^{2}\left(\mathbb{R}_{+}, H_{0}^{1}(] 0, L[)\right)
\end{gathered}
$$

and

$$
\left\{\begin{array}{l}
\eta(x, t, s)=\psi(x, t)-\psi(x, t-s), \\
\eta_{0}(x, s)=\eta(x, 0, s)=\psi_{0}(x, 0)-\psi_{0}(x, s) .
\end{array}\right.
$$

The set $L_{g}^{2}\left(\mathbb{R}_{+}, H_{0}^{1}(] 0, L[)\right)$ is the weighted space with respect to the measure $g(s) d s$ defined by

$$
L_{g}^{2}\left(\mathbb{R}_{+}, H_{0}^{1}(] 0, L[)\right)=\left\{w: \mathbb{R}_{+} \rightarrow H_{0}^{1}(] 0, L[), \quad \int_{0}^{L} \int_{0}^{+\infty} g(s) w_{x}^{2}(x, s) d s d x<+\infty\right\}
$$

and endowed with the classical inner product

$$
\langle v, w\rangle_{L_{g}^{2}\left(\mathbb{R}_{+}, H_{0}^{1}(10, L \mathrm{D})\right)}=\int_{0}^{L} \int_{0}^{+\infty} g(s) v_{x}(x, s) w_{x}(x, s) d s d x .
$$

The operators $\mathscr{B}$ and $\mathscr{A}$ are linear and given by

$$
\mathscr{B}\left(w_{1}, w_{2}, w_{3}, w_{4}, w_{5}\right)^{T}=\left(0,0,0, \frac{\|f\|_{\infty}}{\rho_{2}} w_{4}, \epsilon_{0} w_{5}\right)^{T}
$$

and

$$
\mathscr{A}\left(\begin{array}{c}
w_{1} \\
w_{2} \\
w_{3} \\
w_{4} \\
w_{5}
\end{array}\right)=\left(\begin{array}{c}
w_{3} \\
w_{4} \\
\frac{k_{1}}{\rho_{1}}\left(w_{1 x}+w_{2}\right)_{x} \\
\tilde{w}_{4} \\
-w_{5 s}-\epsilon_{0} w_{5}+w_{4}
\end{array}\right),
$$

where

$$
\begin{gathered}
\tilde{w}_{4}=\frac{1}{\rho_{2}}\left(k_{2}-g_{0}\right) w_{2 x x}-\frac{k_{1}}{\rho_{2}}\left(w_{1 x}+w_{2}\right)-\frac{\|f\|_{\infty}}{\rho_{2}} w_{4} \\
+\frac{1}{\rho_{2}} \int_{0}^{+\infty} g(s) w_{5 x x}(s) d s-\frac{1}{\rho_{2}} \int_{0}^{+\infty} f(s) w_{5 s}(s) d s, \\
\epsilon_{0}=\frac{\alpha^{2} g_{0} c_{0}}{4\|f\|_{\infty}}
\end{gathered}
$$

and $c_{0}$ is the smallest positive constant satisfying (Poincaré's inequality)

$$
\int_{0}^{L} w^{2}(x) d x \leq c_{0} \int_{0}^{L} w_{x}^{2}(x) d x, \quad \forall w \in H_{0}^{1}(] 0, L[)
$$


( $\epsilon_{0}$ is well defined positive constant, since $\|f\|_{\infty}>0$. Otherwise, $f \equiv 0$ and then no distributed time delay is considered in (1.1), which is a well studied case in the literature; see the Introduction). The domains $\mathscr{D}(\mathscr{B})$ and $\mathscr{D}(\mathscr{A})$ of $\mathscr{B}$ and $\mathscr{A}$, respectively, are given by $\mathscr{D}(\mathscr{B})=\mathscr{H}$ and

$$
\mathscr{D}(\mathscr{A})=\left\{\begin{array}{c}
W=\left(w_{1}, w_{2}, w_{3}, w_{4}, w_{5}\right)^{T} \in \mathscr{H}, w_{5}(0)=0 \\
w_{5 s} \in L_{g}^{2}\left(\mathbb{R}_{+}, H_{0}^{1}(] 0, L[)\right), w_{3}, w_{4} \in H_{0}^{1}(] 0, L[), w_{1} \in H^{2}(] 0, L[) \\
\left(k_{2}-g_{0}\right) w_{2 x x}+\int_{0}^{+\infty} g(s) w_{5 x x}(s) d s \in L^{2}(] 0, L[)
\end{array}\right\},
$$

since, thanks to the Cauchy-Schwarz inequality and the first inequality in (2.3),

$$
w_{5 s} \in L_{g}^{2}\left(\mathbb{R}_{+}, H_{0}^{1}(] 0, L[)\right) \Longrightarrow \int_{0}^{+\infty} f(s) w_{5 s}(s) d s \in L^{2}(] 0, L[) .
$$

We use the classical notations $\mathscr{D}\left(\mathscr{A}^{0}\right)=\mathscr{H}, \mathscr{D}\left(\mathscr{A}^{1}\right)=\mathscr{D}(\mathscr{A})$ and

$$
\mathscr{D}\left(\mathscr{A}^{n}\right)=\left\{W \in \mathscr{D}\left(\mathscr{A}^{n-1}\right), \mathscr{A} W \in \mathscr{D}\left(\mathscr{A}^{n-1}\right)\right\}, \quad n=2,3, \cdots,
$$

endowed with the classical graph norm

$$
\|W\|_{\mathscr{D}\left(\mathscr{A}^{n}\right)}=\sum_{k=0}^{n}\left\|\mathscr{A}^{k} W\right\|_{\mathscr{H}} .
$$

On the other hand, keeping in mind the definition of $\eta$ in (2.5), we have

$$
\left\{\begin{array}{l}
\eta_{t}(x, t, s)+\eta_{s}(x, t, s)=\psi_{t}(x, t), \\
\eta(0, t, s)=\eta(L, t, s)=0 \\
\eta(x, t, 0)=0
\end{array}\right.
$$

Therefore, we conclude from (2.6), (2.7), and (2.13) and the equality

$$
\eta_{s}(x, t, s)=\psi_{t}(x, t-s)
$$

that the systems (1.1) and (2.4) are equivalent.

Thanks to (2.1), it is well-known that $\mathscr{H}$ endowed with the inner product, for $W=$ $\left(w_{1}, w_{2}, w_{3}, w_{4}, w_{5}\right)^{T}$ and $\tilde{W}=\left(\tilde{w}_{1}, \tilde{w}_{2}, \tilde{w}_{3}, \tilde{w}_{4}, \tilde{w}_{5}\right)^{T}$,

$$
\begin{aligned}
\langle W, \tilde{W}\rangle_{\mathscr{H}}= & \int_{0}^{L}\left(\left(k_{2}-g_{0}\right) w_{2 x}(x) \tilde{w}_{2 x}(x)+k_{1}\left(w_{1 x}(x)+w_{2}(x)\right)\left(\tilde{w}_{1 x}(x)+\tilde{w}_{2}(x)\right)\right) d x \\
& +\int_{0}^{L}\left(\rho_{1} w_{3}(x) \tilde{w}_{3}(x)+\rho_{2} w_{4}(x) \tilde{w}_{4}(x)\right) d x+\left\langle w_{5}, \tilde{w}_{5}\right\rangle_{L_{g}^{2}\left(\mathbb{R}_{+}, H_{0}^{1}(] 0, L[)\right)}
\end{aligned}
$$

is a Hilbert space and $\mathscr{D}(\mathscr{A}) \subset \mathscr{H}$ with dense embedding, since, using contradiction arguments, this inner product generates on $\mathscr{H}$ a norm equivalent to the one of

$$
\mathscr{H}_{0}:=\left(H^{1}(] 0, L[)\right)^{2} \times\left(L^{2}(] 0, L[)\right)^{2} \times L_{g}^{2}\left(\mathbb{R}_{+}, H^{1}(] 0, L[)\right) ;
$$

that is, there exist two positive constants $d_{1}$ and $d_{2}$ satisfying, for all $W \in \mathscr{H}$,

$$
d_{1}\|W\|_{\mathscr{H}_{0}} \leq\|W\|_{\mathscr{H}} \leq d_{2}\|W\|_{\mathscr{H}_{0}} .
$$

The well-posedness of problem (2.4) is ensured by the following theorem:

Theorem 2.1. Assume that (A1)-(A2) hold. Then, for any $n \in \mathbb{N}$ and $\mathscr{U}_{0} \in \mathscr{D}\left(\mathscr{A}^{n}\right)$, the system (2.4) has a unique solution satisfying

$$
\mathscr{U} \in \cap_{k=0}^{n} C^{k}\left(\mathbb{R}_{+}, \mathscr{D}\left(\mathscr{A}^{n-k}\right)\right)
$$

Proof. To prove Theorem 2.1, we use the semigroup approach. So, first, we show that the linear operator $\mathscr{A}$ is dissipative. Indeed, let $W=\left(w_{1}, w_{2}, w_{3}, w_{4}, w_{5}\right)^{T} \in \mathscr{D}(\mathscr{A})$, then 


$$
\begin{gathered}
\langle\mathscr{A} W, W\rangle_{\mathscr{H}} \\
=\int_{0}^{L}\left(\left(k_{2}-g_{0}\right) w_{4 x}(x) w_{2 x}(x)+k_{1}\left(w_{3 x}(x)+w_{4}(x)\right)\left(w_{1 x}(x)+w_{2}(x)\right)\right) d x \\
+\int_{0}^{L}\left(k_{1}\left(w_{1 x}(x)+w_{2}(x)\right)_{x} w_{3}(x)-k_{1}\left(w_{1 x}(x)+w_{2}(x)\right) w_{4}(x)\right) d x \\
+\int_{0}^{L}\left(\left(k_{2}-g_{0}\right) w_{2 x x}-\|f\|_{\infty} w_{4}(x)\right) w_{4}(x) d x \\
+\int_{0}^{L}\left(\int_{0}^{+\infty} g(s) w_{5 x x}(x, s) d s-\int_{0}^{+\infty} f(s) w_{5 s}(x, s) d s\right) w_{4}(x) d x \\
+\int_{0}^{L} \int_{0}^{+\infty} g(s)\left(-w_{5 s}(x, s)-\epsilon_{0} w_{5}(x, s)+w_{4}(x)\right)_{x} w_{5 x}(x, s) d s d x .
\end{gathered}
$$

It is clear that by integrating by parts with respect to $s$ and using the fact that

$$
\lim _{s \rightarrow+\infty} g(s) w_{5 x}(x, s)=\lim _{s \rightarrow+\infty} f(s) w_{5}(x, s)=0
$$

(due to (A1), (2.3) and (2.9)) and $w_{5}(x, 0)=0($ definition of $\mathscr{D}(\mathscr{A}))$, we find

$$
-\int_{0}^{L} \int_{0}^{+\infty} g(s) w_{5 x}(x, s) w_{5 x s}(x, s) d s d x=\frac{1}{2} \int_{0}^{L} \int_{0}^{+\infty} g^{\prime}(s) w_{5 x}^{2}(x, s) d s d x .
$$

Note that, thanks to (2.2) and the fact that $g$ is nonincreasing and $w_{5} \in L_{g}^{2}\left(\mathbb{R}_{+}, H_{0}^{1}(] 0, L[)\right)$,

$$
\begin{aligned}
\left|\int_{0}^{L} \int_{0}^{+\infty} g^{\prime}(s) w_{5 x}^{2}(x, s) d s\right| & =-\int_{0}^{L} \int_{0}^{+\infty} g^{\prime}(s) w_{5 x}^{2}(x, s) d s \\
& \leq \theta_{0} \int_{0}^{L} \int_{0}^{+\infty} g(s) w_{5 x}^{2}(x, s) d s \\
& <+\infty
\end{aligned}
$$

so the integral in the right hand side of (2.18) is well defined. Moreover,

$$
-\int_{0}^{L} w_{4}(x) \int_{0}^{+\infty} f(s) w_{5 s}(x, s) d s d x=\int_{0}^{L} w_{4}(x) \int_{0}^{+\infty} f^{\prime}(s) w_{5}(x, s) d s d x .
$$

Consequently, inserting (2.18) and (2.19) in (2.17) and integrating by parts with respect to $x$, we get

$$
\begin{aligned}
\langle\mathscr{A} W, W\rangle_{\mathscr{H}}= & \frac{1}{2} \int_{0}^{L} \int_{0}^{+\infty} g^{\prime}(s) w_{5 x}^{2}(x, s) d s d x \\
& +\int_{0}^{L} w_{4}(x) \int_{0}^{+\infty} f^{\prime}(s) w_{5}(x, s) d s d x \\
& -\|f\|_{\infty} \int_{0}^{L} w_{4}^{2}(x) d x-\epsilon_{0} \int_{0}^{L} \int_{0}^{+\infty} g(s) w_{5 x}^{2}(x, s) d s d x .
\end{aligned}
$$

Now, using Young's inequalities, (2.9) and the second inequality in (2.3) imply that ( $\epsilon_{0}$ and $c_{0}$ are defined, respectively, in (2.8) and (2.9)),

$$
\int_{0}^{L} w_{4}(x) \int_{0}^{+\infty} f^{\prime}(s) w_{5}(x, s) d s d x
$$




$$
\begin{aligned}
& \leq \frac{\alpha}{2} \sqrt{\frac{c_{0}\|f\|_{\infty}}{\epsilon_{0} g_{0}}} \int_{0}^{L} \int_{0}^{+\infty} g(s) w_{4}^{2}(x) d s d x+\frac{\alpha}{2} \sqrt{\frac{\epsilon_{0} g_{0}}{c_{0}\|f\|_{\infty}}} \int_{0}^{L} \int_{0}^{+\infty} g(s) w_{5}^{2}(x, s) d s d x \\
& \leq \frac{\alpha}{2} \sqrt{\frac{c_{0} g_{0}\|f\|_{\infty}}{\epsilon_{0}}} \int_{0}^{L} w_{4}^{2}(x) d x+\frac{\alpha}{2} \sqrt{\frac{\epsilon_{0} g_{0} c_{0}}{\|f\|_{\infty}}} \int_{0}^{L} \int_{0}^{+\infty} g(s) w_{5 x}^{2}(x, s) d s d x \\
& \leq\|f\|_{\infty} \int_{0}^{L} w_{4}^{2}(x) d x+\epsilon_{0} \int_{0}^{L} \int_{0}^{+\infty} g(s) w_{5 x}^{2}(x, s) d s d x .
\end{aligned}
$$

Finally, combining (2.20) and (2.21), and using the fact that $g$ is nonincreasing, we obtain

$$
\langle\mathscr{A} W, W\rangle_{\mathscr{H}} \leq \frac{1}{2} \int_{0}^{L} \int_{0}^{+\infty} g^{\prime}(s) w_{5 x}^{2}(x, s) d s d x \leq 0,
$$

which means that $\mathscr{A}$ is dissipative.

Next, we shall prove that $I d-\mathscr{A}$ is surjective. Indeed, let $F=\left(f_{1}, f_{2}, f_{3}, f_{4}, f_{5}\right)^{T} \in \mathscr{H}$, we show that there exists $W=\left(w_{1}, w_{2}, w_{3}, w_{4}, w_{5}\right)^{T} \in \mathscr{D}(\mathscr{A})$ satisfying

$$
(I d-\mathscr{A}) W=F,
$$

which is equivalent to

$$
\left\{\begin{array}{l}
w_{3}=w_{1}-f_{1}, \\
w_{4}=w_{2}-f_{2}, \\
\rho_{1} w_{1}-k_{1}\left(w_{1 x}+w_{2}\right)_{x}=\rho_{1}\left(f_{1}+f_{3}\right), \\
\quad\left(\rho_{2}+\|f\|_{\infty}\right) w_{2}-\left(k_{2}-g_{0}\right) w_{2 x x}+k_{1}\left(w_{1 x}+w_{2}\right)-\int_{0}^{+\infty} g(s) w_{5 x x}(s) d s \\
\quad \quad+\int_{0}^{+\infty} f(s) w_{5 s}(s) d s=\left(\rho_{2}+\|f\|_{\infty}\right) f_{2}+\rho_{2} f_{4}, \\
\quad w_{5 s}+\left(1+\epsilon_{0}\right) w_{5}=w_{2}+f_{5}-f_{2} .
\end{array}\right.
$$

We note that the last equation in (2.24) with $w_{5}(0)=0$ has the unique solution

$$
\begin{aligned}
w_{5}(s) & =e^{-\left(1+\epsilon_{0}\right) s} \int_{0}^{s} e^{\left(1+\epsilon_{0}\right) y}\left(w_{2}+f_{5}(y)-f_{2}\right) d y \\
& =\left(1+\epsilon_{0}\right)^{-1}\left(1-e^{-\left(1+\epsilon_{0}\right) s}\right) w_{2}+e^{-\left(1+\epsilon_{0}\right) s} \int_{0}^{s} e^{\left(1+\epsilon_{0}\right) y}\left(f_{5}(y)-f_{2}\right) d y .
\end{aligned}
$$

Next, plugging (2.25) into the fourth equation in (2.24), we get

$$
\left\{\begin{array}{l}
\rho_{1} w_{1}-k_{1}\left(w_{1 x}+w_{2}\right)_{x}=\rho_{1}\left(f_{1}+f_{3}\right), \\
l_{2} w_{2}-l_{1} w_{2 x x}+k_{1}\left(w_{1 x}+w_{2}\right)=\tilde{f},
\end{array}\right.
$$

where

$$
\begin{gathered}
l_{1}=k_{2}-g_{0}+\left(1+\epsilon_{0}\right)^{-1} g_{0}-\left(1+\epsilon_{0}\right)^{-1} \int_{0}^{+\infty} g(s) e^{-\left(1+\epsilon_{0}\right) s} d s, \\
l_{2}=\rho_{2}+\|f\|_{\infty}+\int_{0}^{+\infty} f(s) e^{-\left(1+\epsilon_{0}\right) s} d s
\end{gathered}
$$

and

$$
\begin{aligned}
\tilde{f}= & \left(\rho_{2}+\|f\|_{\infty}\right) f_{2}+\rho_{2} f_{4}+\left(1+\epsilon_{0}\right) \int_{0}^{+\infty} f(s) e^{-\left(1+\epsilon_{0}\right) s}\left(\int_{0}^{s} e^{\left(1+\epsilon_{0}\right) y}\left(f_{5}(y)-f_{2}\right) d y\right) d s \\
& -\int_{0}^{+\infty} f(s)\left(f_{5}(s)-f_{2}\right) d s+\int_{0}^{+\infty} g(s) e^{-\left(1+\epsilon_{0}\right) s}\left(\int_{0}^{s} e^{\left(1+\epsilon_{0}\right) y}\left(f_{5}(y)-f_{2}\right)_{x x} d y\right) d s .
\end{aligned}
$$


It remains only to prove that $(2.26)$ has a solution $\left(w_{1}, w_{2}\right) \in\left(H_{0}^{1}(] 0, L[)\right)^{2}$. Then, substituting in (2.25) and the first two equations in (2.24), we obtain $W \in \mathscr{D}(\mathscr{A})$ satisfying (2.23). Since $l_{1} \geq k_{2}$ $-g_{0}>0$ (according to (2.1)) and

$$
l_{2} \geq \rho_{2}+\|f\|_{\infty}-\|f\|_{\infty} \frac{1}{1+\epsilon_{0}} \geq \rho_{2}>0
$$

we see that the operator

$$
\mathscr{K}\left(\begin{array}{l}
w_{1} \\
w_{2}
\end{array}\right)=\left(\begin{array}{c}
\rho_{1} w_{1}-k_{1}\left(w_{1 x}+w_{2}\right)_{x} \\
l_{2} w_{2}-l_{1} w_{2 x x}+k_{1}\left(w_{1 x}+w_{2}\right)
\end{array}\right),
$$

is self-adjoint linear positive definite. Considering the variational formulation of (2.26), and applying the Lax-Milgram theorem and classical regularity arguments, we conclude that (2.26) has a unique solution $\left(w_{1}, w_{2}\right) \in\left(H_{0}^{1}(] 0, L[)\right)^{2}$ satisfying the third and fourth equations of (2.24), since (2.25). Therefore, using (2.11),

$$
\left(k_{2}-g_{0}\right) w_{2 x x}+\int_{0}^{+\infty} g(s) w_{5 x x}(s) d s \in L^{2}(] 0, L[) .
$$

This proves that $I d-\mathscr{A}$ is surjective. Finally, we note that (2.22) and (2.23) mean that $-\mathscr{A}$ is a maximal monotone operator. Hence, using Lummer-Phillips theorem (see Ref. 34), we deduce that $\mathscr{A}$ is an infinitesimal generator of a linear $C_{0}$-semigroup on $\mathscr{H}$.

On the other hand, as the linear operator $\mathscr{B}$ (defined in (2.6)) is Lipschitz continuous, it follows that $\mathscr{A}+\mathscr{B}$ also is an infinitesimal generator of a linear $C_{0}$-semigroup on $\mathscr{H}$ (see Ref. 34: Chap. 3 - Theorem 1.1). Consequently, (2.4) is well-posed in the sense of Theorem 2.1 (see Refs. 22 and 34).

\section{ASYMPTOTIC STABILITY}

In this section, we investigate the asymptotic behavior of the solution of (2.4) by the use of the energy method. We produce suitable Lyapunov functionals and prove some decay estimates depending on the asymptotic behavior of $g$, the connection between $g$ and $f$, and the regularity of initial data.

\section{A. Additional assumptions and stability results}

Our asymptotic stability results hold under the following additional assumptions:

(A3) The function $g$ satisfies

$$
g_{0}:=\int_{0}^{+\infty} g(s) d s>0
$$

and one of the following two conditions holds:

$$
\exists \theta_{1}>0, g^{\prime}(s) \leq-\theta_{1} g(s), \quad \forall s \in \mathbb{R}_{+}
$$

or there exists a positive nonincreasing function $\xi: \mathbb{R}_{+} \rightarrow \mathbb{R}_{+}^{*}$ of class $C\left(\mathbb{R}_{+}, \mathbb{R}_{+}^{*}\right)$ such that

$$
\begin{cases}g(t-s) \geq \xi(t) \int_{t}^{+\infty} g(\tau-s) d \tau, & \forall t \in \mathbb{R}_{+}, \quad \forall s \in[0, t], \\ g^{\prime}(s)<0, & \forall s \in \mathbb{R}_{+} .\end{cases}
$$

(A4) There exists a positive even function $\gamma: \mathbb{R} \rightarrow \mathbb{R}_{+}^{*}$ of class $C\left(\mathbb{R}, \mathbb{R}_{+}^{*}\right)$ and nonincreasing on $\mathbb{R}_{+}$, and a positive function $\beta: \mathbb{R}_{+} \rightarrow \mathbb{R}_{+}^{*}$ of class $C\left(\mathbb{R}_{+}, \mathbb{R}_{+}^{*}\right)$ such that

$$
\beta_{0}:=\int_{0}^{+\infty} \beta(s) d s<+\infty
$$


and

$$
|f(s)| \leq e^{-\tilde{\gamma}(s)} \beta(s) g(s), \quad \forall s \in \mathbb{R}_{+},
$$

where

$$
\tilde{\gamma}(s)=2 \int_{0}^{\frac{s}{2}} \gamma(\tau) d \tau, \quad \forall s \in \mathbb{R}_{+} .
$$

Moreover, when (1.2) does not hold, we assume also that $f$ is of class $C^{3}\left(\mathbb{R}_{+}, \mathbb{R}\right)$ and satisfies, for some positive constant $\tilde{\alpha}$,

$$
\left|f^{\prime \prime}(s)\right| \leq \tilde{\alpha} g(s) \quad \text { and } \quad\left|f^{\prime \prime \prime}(s)\right| \leq \tilde{\alpha} g(s), \quad \forall s \in \mathbb{R}_{+} .
$$

Remark 3.1. The condition (2.2) implies that the decay rate of $g$ is at most of exponential type. The conditions (3.2) and (3.3) include, respectively, the class of functions $g$ which converge to zero at least exponentially or less than exponentially. When

$$
\lim _{t \rightarrow+\infty} \xi(t)>0
$$

the first inequality in (3.3), introduced in Refs. 39 and 42, implies that g converges to zero at least exponentially but it does not involve the derivative of $g$. We distinguish the cases (3.2) and (3.3) because they lead to different kinds of decays.

Theorem 3.2. Assume that (A1)-(A4) hold. Then there exists a positive constant $\delta_{0}$ independent off such that, if

$$
\int_{0}^{+\infty}|f(s)| d s<\delta_{0}
$$

then, we have the following stability results:

(i) Equal speed propagation and exponential decay of $g$ : if (1.2) and (3.2) hold, then, for any $\mathscr{U}_{0} \in \mathscr{D}(\mathscr{A})$, there exist positive constants $\delta_{1}$ and $\delta_{2}$ such that

$$
\|\mathscr{U}(t)\|_{\mathscr{H}}^{2} \leq \delta_{2} e^{-\delta_{1} \phi(t)}, \quad \forall t \in \mathbb{R}_{+},
$$

where

$$
\phi(t)=\int_{0}^{t} \min \{1, \gamma(s)\} d s
$$

(ii) Nonequal speed propagation and exponential decay of $g$ : if (1.2) does not hold and (3.2) holds, then, for any $n=2,3, \cdots$ and $\mathscr{U}_{0} \in \mathscr{D}\left(\mathscr{A}^{n}\right)$, there exists a positive constant $\delta_{1}$ such that

$$
\|\mathscr{U}(t)\|_{\mathscr{H}}^{2} \leq \frac{\delta_{1}}{(1+t)^{n-1}}, \quad \forall t \in \mathbb{R}_{+} .
$$

(iii) Equal speed propagation and arbitrary decay of $g$ : if (3.2) does not hold, and (1.2) and (3.3) hold, then, for any $\mathscr{U}_{0} \in \mathscr{D}(\mathscr{A})$, there exist positive constants $\delta_{1}$ and $\delta_{2}$ such that

$$
\|\mathscr{U}(t)\|_{\mathscr{H}}^{2} \leq \delta_{2} e^{-\delta_{1} \phi(t)}\left(1+\int_{0}^{L} \int_{0}^{t} e^{\delta_{1} \phi(s)} \int_{s}^{+\infty} g(\tau) \psi_{0 x}^{2}(x, \tau-s) d \tau d s d x\right), \forall t \in \mathbb{R}_{+},
$$

where

$$
\phi(t)=\int_{0}^{t} \min \{1, \gamma(s), \xi(s)\} d s
$$




\section{B. Examples and comments}

Let us illustrate our decay estimates (3.9), (3.11), and (3.12) by the following simple examples (some of them were given in Ref. 20 for (1.5)):

\section{Equal speed propagation and exponential decay of g: (1.2) and (3.2) hold}

Let us consider the class $g(s)=\alpha_{2} e^{-\alpha_{1} s}$, with $\alpha_{1}, \alpha_{2}>0$. Then (A1) and (3.1) hold provided that $\alpha_{2}$ is small enough so that (2.1) holds. This class satisfies (3.2) with $\theta_{1}=\alpha_{1}$.

3.2.1.1. If

$$
|f(s)| \leq \beta_{2} e^{-\beta_{1}(s+1)^{p}} g(s), \quad \forall s \in \mathbb{R}_{+},
$$

for some constants $\beta_{1}, \beta_{2}, p>0$ with $\beta_{2}$ small enough so that (3.8) holds, then (3.5) is satisfied with $\beta(s)=\beta_{2} e^{-\beta_{0}(s+1)^{p}}$,

$$
\gamma(s)=q\left(\beta_{1}-\beta_{0}\right)(2|s|+1)^{q-1},
$$

any $\left.\beta_{0} \in\right] 0, \beta_{1}$ [ and $q=\min \{p, 1\}$ (so $\gamma$ is positive on $\mathbb{R}$ and nonincreasing on $\mathbb{R}_{+}$), and therefore, (3.9) gives, for some positive constants $c^{\prime}$ and $c^{\prime \prime}$,

$$
\|\mathscr{U}(t)\|_{\mathscr{H}}^{2} \leq c^{\prime \prime} e^{-c^{\prime}(t+1)^{q}}, \quad \forall t \in \mathbb{R}_{+} .
$$

\subsubsection{If}

$$
|f(s)| \leq \beta_{2} e^{-\beta_{1}(\ln (s+1))^{p}} g(s), \quad \forall s \in \mathbb{R}_{+},
$$

for some constants $\beta_{1}, \beta_{2}>0$, and $p>1$ with $\beta_{2}$ small enough so that (3.8) holds, then (3.5) holds with $\beta(s)=\beta_{3} e^{-\beta_{0}(\ln (s+1))^{p}}$,

$$
\gamma(s)= \begin{cases}p\left(\beta_{1}-\beta_{0}\right) \frac{(\ln (2|s|+1))^{p-1}}{2|s|+1} & \text { if }|s| \geq \frac{1}{2}\left(e^{p-1}-1\right):=s_{0}, \\ p\left(\beta_{1}-\beta_{0}\right)(p-1)^{p-1} e^{1-p}:=\tilde{c} & \text { if }|s| \in\left[0, s_{0}\right],\end{cases}
$$

$\beta_{3}=\beta_{2} e^{2 \tilde{c} s_{0}}$ and any $\left.\beta_{0} \in\right] 0, \beta_{1}\left[\right.$ (so $\gamma$ is positive and continuous on $\mathbb{R}$, and nonincreasing on $\mathbb{R}_{+}$), and therefore, (3.9) gives, for some positive constants $c^{\prime}$ and $c^{\prime \prime}$,

$$
\|\mathscr{U}(t)\|_{\mathscr{H}}^{2} \leq c^{\prime \prime} e^{-c^{\prime}(\ln (t+1))^{p}}, \quad \forall t \in \mathbb{R}_{+} .
$$

3.2.1.3. If

$$
|f(s)| \leq \frac{\beta_{1}}{(s+1)^{p}} g(s), \quad \forall s \in \mathbb{R}_{+},
$$

for some constants $\beta_{1}>0$ and $p>1$ with $\beta_{1}$ small enough so that (3.8) holds, then (3.5) holds with $\beta(s)=\frac{\beta_{1}}{(s+1)^{p-\beta_{0}}}$,

$$
\gamma(s)=\frac{\beta_{0}}{2|s|+1}
$$

and any $\left.\beta_{0} \in\right] 0, p-1\left[\right.$ (so $\beta$ is integrable on $\mathbb{R}_{+}$), and therefore, (3.9) gives, for some positive constants $c^{\prime}$ and $c^{\prime \prime}$,

$$
\|\mathscr{U}(t)\|_{\mathscr{H}}^{2} \leq c^{\prime \prime}(t+1)^{-c^{\prime}}, \quad \forall t \in \mathbb{R}_{+} .
$$

\section{Nonequal speed propagation and exponential decay of g: (1.2) does not hold and (3.2) holds}

The estimate (3.11) gives a decay rate of polynomial type for the solution of (2.4), where the decay rate depends on the regularity of initial data $\mathscr{U}_{0}$. 


\section{Equal speed propagation and arbitrary decay of g: (3.2) does not hold, and (1.2) and (3.3) hold}

Let us consider the classes (3.14), (3.16), and (3.18) of $f$, and the following three classes of $g$ which satisfy (3.3) and do not satisfy (3.2).

3.2.3.1. If

$$
g(s)=\alpha_{2}(s+1)^{r-1} e^{-\alpha_{1}(s+1)^{r}},
$$

for some constants $\alpha_{1}, \alpha_{2}>0$, and $r \in$ ]0, 1[. Then (A1) and (3.1) hold provided that $\alpha_{2}$ is small enough so that (2.1) holds. On the other hand, (3.3) holds with $\xi(s)=\alpha_{1}(s+1)^{r-1}$, and therefore, (3.12) holds with

$$
\phi(t)= \begin{cases}\int_{0}^{t} \xi(s) d s & \text { in case (3.14) with } r \leq p, \\ \int_{0}^{t} \gamma(s) d s & \text { in case (3.14) with } r>p, \text { and in cases (3.16) and (3.18). }\end{cases}
$$

If, for example, for some positive constants $\lambda_{0}$ and $M_{0}$,

$$
\int_{0}^{L} \psi_{0 x}^{2}(x, s) d x \leq M_{0} e^{(\ln (s+1))^{\lambda_{0}}}, \quad \forall s \in \mathbb{R}_{+},
$$

then (3.12) implies that, for some positive constants $c^{\prime}$ and $c^{\prime \prime}$, and for all $t \in \mathbb{R}_{+}$,

$$
\|\mathscr{U}(t)\|_{\mathscr{H}}^{2} \leq \begin{cases}c^{\prime \prime} e^{-c^{\prime}(t+1)^{\min \{p, r\}}} & \text { in case (3.14), } \\ c^{\prime \prime} e^{-c^{\prime}(\ln (t+1))^{p}} & \text { in case (3.16), } \\ c^{\prime \prime}(t+1)^{-c^{\prime}} & \text { in case (3.18). }\end{cases}
$$

3.2.3.2. If

$$
g(s)=\frac{\alpha_{2}}{s+e^{r-1}}\left(\ln \left(s+e^{r-1}\right)\right)^{r-1} e^{-\alpha_{1}\left(\ln \left(s+e^{r-1}\right)\right)^{r}},
$$

for some constants $\alpha_{1}, \alpha_{2}>0$, and $r>1$. Then (A1) and (3.1) hold provided that $\alpha_{2}$ is small enough so that (2.1) holds. On the other hand, (3.3) holds with $\xi(s)=r \alpha_{1}\left(s+e^{r-1}\right)^{-1}(\ln (s+$ $\left.\left.e^{r-1}\right)\right)^{r-1}$, and therefore, (3.12) holds with

$$
\phi(t)= \begin{cases}\int_{0}^{t} \xi(s) d s & \text { in case (3.14), and in case (3.16) with } r \leq p, \\ \int_{0}^{t} \gamma(s) d s & \text { in case (3.18), and in case (3.16) with } r>p .\end{cases}
$$

If, for example, for some positive constants $\lambda_{0}$ and $M_{0}$,

$$
\int_{0}^{L} \psi_{0 x}^{2}(x, s) d x \leq M_{0}(s+1)^{\lambda_{0}}, \quad \forall s \in \mathbb{R}_{+},
$$

then (3.12) implies that, for some positive constants $c^{\prime}$ and $c^{\prime \prime}$, and for all $t \in \mathbb{R}_{+}$,

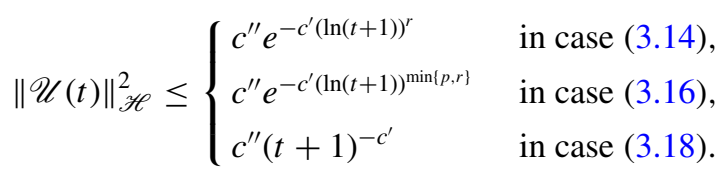

3.2.3.3. If

$$
g(s)=\alpha_{1}(s+1)^{-r},
$$

for some constants $\alpha_{1}>0$ and $r>1$. Then (A1) and (3.1) hold provided that $\alpha_{1}$ is small enough so that (2.1) holds. On the other hand, (3.3) is satisfied with $\xi(s)=(r-1)(s+1)^{-1}$, and therefore, (3.12) holds with $\phi(t)=\int_{0}^{t} \xi(s) d s$. 
If, for example, for some positive constants $\lambda_{0}$ and $M_{0}$,

$$
\int_{0}^{L} \psi_{0 x}^{2}(x, s) d x \leq M_{0}(\ln (s+2))^{\lambda_{0}}, \quad \forall s \in \mathbb{R}_{+}, \quad \forall s \in \mathbb{R}_{+},
$$

then (3.12) implies that, for some positive constants $c^{\prime}$ and $c^{\prime \prime}$, and for all $t \in \mathbb{R}_{+}$,

$$
\|\mathscr{U}(t)\|_{\mathscr{H}}^{2} \leq \begin{cases}c^{\prime \prime}(t+1)^{-c^{\prime}} & \text { if } r>2 \\ c^{\prime \prime}(t+1)^{c^{\prime}} & \text { if } r \leq 2\end{cases}
$$

The estimate (3.28) in case $1<r \leq 2$ does not imply the strong stability of (2.4),

$$
\lim _{t \rightarrow+\infty}\|\mathscr{U}(t)\|_{\mathscr{H}}^{2}=0
$$

The $\lim _{t \rightarrow+\infty}\|\mathscr{U}(t)\|_{\mathscr{H}}^{2}$ in (3.12) depends on the connection between the growths at infinity of $g, f$, and $\int_{0}^{L} \psi_{0 x}^{2}(x, \cdot) d x$

\section{Proof of Theorem 3.2}

We start our proof by giving the modified energy functional $E$ associated with any weak solution of (2.4) (corresponding to initial data $\mathscr{U}_{0} \in \mathscr{H}$ ),

$$
E(t):=\frac{1}{2}\|\mathscr{U}(t)\|_{\mathscr{H}}^{2}
$$

Let $\mathscr{U}_{0} \in \mathscr{D}\left(\mathscr{A}^{n_{0}}\right)$, where $n_{0}=1$ if (1.2) holds, and $n_{0}=2$ if (1.2) does not hold, so that all the calculations below are justified. From (2.4), (2.6), (2.14), (2.19), and (2.20) we get

$$
E^{\prime}(t)=\frac{1}{2} \int_{0}^{L} \int_{0}^{+\infty} g^{\prime}(s) \eta_{x}^{2}(x, t, s) d s d x-\int_{0}^{L} \psi_{t}(x, t) \int_{0}^{+\infty} f(s) \psi_{t}(x, t-s) d s d x
$$

Note that, in contrast to the situation of absence of delay and/or presence of frictional damping considered in the literature (as in (1.3)), we are unable to determine the sign of $E^{\prime}$ from (3.31), and therefore, the system (2.4) is not necessarily dissipative with respect to $E$ at this stage.

On the other hand, using Cauchy-Schwarz inequality, the following classical inequalities hold, for all $v \in C\left(\mathbb{R}_{+}, \mathbb{R}_{+}\right)$(see, for example, Refs. 16 and 26),

$$
\left(\int_{0}^{+\infty} g(s) v(s) d s\right)^{2} \leq g_{0} \int_{0}^{+\infty} g(s) v^{2}(s) d s
$$

and

$$
\left(\int_{0}^{+\infty} g^{\prime}(s) v(s) d s\right)^{2} \leq-g(0) \int_{0}^{+\infty} g^{\prime}(s) v^{2}(s) d s .
$$

Inequalities (3.32) and (3.33) will be repeatedly used in the proof. Also, we will denote by $c_{\delta}$ a positive constant depending on some parameter $\delta$.

In order to prove (3.9), (3.11), and (3.12) we prove briefly several lemmas. Lemmas 3.3-3.9 and 3.11 are known in case $f \equiv 0$ (see, for example, Refs. 2,8,17,19,25, and 26), while the ones 3.10 and 3.12-3.14 are introduced in the present paper to cope with the new situation due to the distributed time delay and the nondissipativeness character of (1.1).

Lemma 3.3. The functional

$$
I_{1}(t):=-\rho_{2} \int_{0}^{L} \psi_{t}(x, t) \int_{0}^{+\infty} g(s) \eta(x, t, s) d s d x,
$$


satisfies, for all $\delta>0$,

$$
\begin{aligned}
I_{1}^{\prime}(t) \leq & \int_{0}^{L}\left(-\rho_{2}\left(g_{0}-\delta\right) \psi_{t}^{2}(x, t)+\delta\left(\psi_{x}^{2}(x, t)+\left(\varphi_{x}(x, t)+\psi(x, t)\right)^{2}\right)\right) d x \\
& +c_{\delta} \int_{0}^{L} \int_{0}^{+\infty}\left(g(s) \eta_{x}^{2}(x, t, s)-g^{\prime}(s) \eta_{x}^{2}(x, t, s)\right) d s d x \\
& +\int_{0}^{L}\left(\int_{0}^{+\infty} f(s) \psi_{t}(x, t-s) d s\right)\left(\int_{0}^{+\infty} g(s) \eta(x, t, s) d s\right) d x .
\end{aligned}
$$

Proof. The proof is identical to the one given in Refs. 25 and 26 in case $f \equiv 0$. Indeed, by differentiating $I_{1}$, using the second equation in (1.1) and the first one in (2.13), and integrating by parts, we get

$$
\begin{aligned}
I_{1}^{\prime}(t)= & -\rho_{2} g_{0} \int_{0}^{L} \psi_{t}^{2}(x, t) d x+\int_{0}^{L}\left(\int_{0}^{+\infty} f(s) \psi_{t}(x, t-s) d s\right)\left(\int_{0}^{+\infty} g(s) \eta(x, t, s) d s\right) d x \\
& +\int_{0}^{L}\left(\int_{0}^{+\infty} g(s) \eta_{x}(x, t, s) d s\right)^{2} d x-g_{0} \int_{0}^{L} \psi_{x}(x, t) \int_{0}^{+\infty} g(s) \eta_{x}(x, t, s) d s d x \\
& -\rho_{2} \int_{0}^{L} \psi_{t}(x, t) \int_{0}^{+\infty} g^{\prime}(s) \eta(x, t, s) d s d x+k_{2} \int_{0}^{L} \psi_{x}(x, t) \int_{0}^{+\infty} g(s) \eta_{x}(x, t, s) d s d x \\
& +k_{1} \int_{0}^{L}\left(\varphi_{x}(x, t)+\psi(x, t)\right) \int_{0}^{+\infty} g(s) \eta(x, t, s) d s d x
\end{aligned}
$$

Applying Cauchy-Schwarz inequality, (2.9) and Young's inequality

$$
a b \leq \frac{d}{2} a^{2}+\frac{1}{2 d} b^{2}, \quad \forall a, b \in \mathbb{R}, \quad \forall d>0,
$$

to the last four terms of the above equality, and using (3.33), we get

$$
\begin{aligned}
I_{1}^{\prime}(t) \leq & \int_{0}^{L}\left(-\rho_{2}\left(g_{0}-\delta\right) \psi_{t}^{2}(x, t)+\frac{\delta}{2} \psi_{x}^{2}(x, t)+\delta\left(\varphi_{x}(x, t)+\psi(x, t)\right)^{2}\right) d x \\
& +\int_{0}^{L}\left(\int_{0}^{+\infty} f(s) \psi_{t}(x, t-s) d s\right)\left(\int_{0}^{+\infty} g(s) \eta(x, t, s) d s\right) d x \\
& -c_{\delta} \int_{0}^{L} \int_{0}^{+\infty} g^{\prime}(t) \eta_{x}^{2}(x, t, s) d s d x+c_{\delta} \int_{0}^{L}\left(\int_{0}^{+\infty} g(t) \eta_{x}(x, t, s) d s\right)^{2} d x \\
& -g_{0} \int_{0}^{L} \psi_{x}(x, t) \int_{0}^{+\infty} g(s) \eta_{x}(x, t, s) d s d x .
\end{aligned}
$$

Again, applying Cauchy-Schwarz and Young's inequalities to the last term in (3.35), and using (3.32), we find (3.34).

In case (3.3), we will consider another manipulations for the last two terms in (3.35).

Lemma 3.4. The functional

$$
I_{2}(t):=-\int_{0}^{L}\left(\rho_{1} \varphi(x, t) \varphi_{t}(x, t)+\rho_{2} \psi(x, t) \psi_{t}(x, t)\right) d x
$$


satisfies, for some $c_{1}>0$ (not depending on $f$ ),

$$
\begin{aligned}
I_{2}^{\prime}(t) \leq & -\int_{0}^{L}\left(\rho_{1} \varphi_{t}^{2}(x, t)+\rho_{2} \psi_{t}^{2}(x, t)\right) d x+k_{1} \int_{0}^{L}\left(\varphi_{x}(x, t)+\psi(x, t)\right)^{2} d x \\
& +c_{1} \int_{0}^{L} \psi_{x}^{2}(x, t) d x+c_{1} \int_{0}^{L} \int_{0}^{+\infty} g(s) \eta_{x}^{2}(x, t, s) d s d x \\
& +\int_{0}^{L} \psi(x, t) \int_{0}^{+\infty} f(s) \psi_{t}(x, t-s) d s d x .
\end{aligned}
$$

Proof. Similar to the proof of Refs. 2,8,25, and 26 in case $f \equiv 0$, by differentiating $I_{2}$, using the first two equations in (1.1) and integrating by parts, we find

$$
\begin{aligned}
I_{2}^{\prime}(t)= & -\int_{0}^{L}\left(\rho_{1} \varphi_{t}^{2}(x, t)+\rho_{2} \psi_{t}^{2}(x, t)\right) d x+k_{1} \int_{0}^{L}\left(\varphi_{x}(x, t)+\psi(x, t)\right)^{2} d x \\
& +\left(k_{2}-g_{0}\right) \int_{0}^{L} \psi_{x}^{2}(x, t) d x+\int_{0}^{L} \psi(x, t) \int_{0}^{+\infty} f(s) \psi_{t}(x, t-s) d s d x \\
& +\int_{0}^{L} \psi_{x}(x, t) \int_{0}^{+\infty} g(s) \eta_{x}(x, t, s) d s d x
\end{aligned}
$$

The use of Young's inequality and (3.32) for the last term in (3.37) leads to (3.36).

Lemma 3.5. The functional

$$
\begin{aligned}
I_{3}(t):= & \rho_{2} \int_{0}^{L} \psi_{t}(x, t)\left(\varphi_{x}(x, t)+\psi(x, t)\right) d x+\frac{k_{2} \rho_{1}}{k_{1}} \int_{0}^{L} \psi_{x}(x, t) \varphi_{t}(x, t) d x \\
& -\frac{\rho_{1}}{k_{1}} \int_{0}^{L} \varphi_{t}(x, t) \int_{0}^{+\infty} g(s) \psi_{x}(x, t-s) d s d x
\end{aligned}
$$

satisfies, for all $\varepsilon>0$,

$$
\begin{aligned}
I_{3}^{\prime}(t) \leq & \rho_{2} \int_{0}^{L} \psi_{t}^{2}(x, t) d x+\frac{1}{2 \varepsilon}\left(k_{2} \psi_{x}(L, t)-\int_{0}^{+\infty} g(s) \psi_{x}(L, t-s) d s\right)^{2} \\
& +\frac{1}{2 \varepsilon}\left(k_{2} \psi_{x}(0, t)-\int_{0}^{+\infty} g(s) \psi_{x}(0, t-s) d s\right)^{2} \\
& +\frac{\varepsilon}{2}\left(\varphi_{x}^{2}(L, t)+\varphi_{x}^{2}(0, t)\right)-k_{1} \int_{0}^{L}\left(\varphi_{x}(x, t)+\psi(x, t)\right)^{2} d x \\
& -c_{\varepsilon} \int_{0}^{L} \int_{0}^{+\infty} g^{\prime}(s) \eta_{x}^{2}(x, t, s) d s d x+\varepsilon \int_{0}^{L} \varphi_{t}^{2}(x, t) d x \\
& -\int_{0}^{L}\left(\varphi_{x}(x, t)+\psi(x, t)\right) \int_{0}^{+\infty} f(s) \psi_{t}(x, t-s) d s d x \\
& +\left(\frac{\rho_{1} k_{2}}{k_{1}}-\rho_{2}\right) \int_{0}^{L} \varphi_{t}(x, t) \psi_{x t}(x, t) d x .
\end{aligned}
$$

Proof. As in Ref. 2 in case $f \equiv 0$, a simple differentiation of $I_{3}$, using the first two equations in (1.1) and the fact that

$$
\psi_{x t}(x, t-s)=\eta_{x s}(x, t, s)
$$


and integration by parts give

$$
\begin{aligned}
I_{3}^{\prime}(t)= & -k_{1} \int_{0}^{L}\left(\varphi_{x}(x, t)+\psi(x, t)\right)^{2} d x-\frac{\rho_{1}}{k_{1}} \int_{0}^{L} \varphi_{t}(x, t) \int_{0}^{+\infty} g(s) \psi_{x t}(x, t-s) d s d x \\
& +\left(\frac{k_{2} \rho_{1}}{k_{1}}-\rho_{2}\right) \int_{0}^{L} \varphi_{t}(x, t) \psi_{x t}(x, t) d x+k_{2} \int_{0}^{L} \psi_{x}(x, t)\left(\varphi_{x}(x, t)+\psi(x, t)\right)_{x} d x \\
& +\int_{0}^{L}\left(k_{2} \psi_{x x}(x, t)-\int_{0}^{+\infty} g(s) \psi_{x x}(x, t-s) d s\right)\left(\varphi_{x}(x, t)+\psi(x, t)\right) d x \\
& -\int_{0}^{L}\left(\int_{0}^{+\infty} f(s) \psi_{t}(x, t-s) d s\right)\left(\varphi_{x}(x, t)+\psi(x, t)\right) d x \\
& +\rho_{2} \int_{0}^{L} \psi_{t}^{2}(x, t) d x-\int_{0}^{L}\left(\varphi_{x}(x, t)+\psi(x, t)\right)_{x} \int_{0}^{+\infty} g(s) \psi_{x}(x, t-s) d s d x \\
= & -k_{1} \int_{0}^{L}\left(\varphi_{x}(x, t)+\psi(x, t)\right)^{2} d x+\rho_{2} \int_{0}^{L} \psi_{t}^{2}(x, t) d x+\left(\frac{\rho_{1} k_{2}}{k_{1}}-\rho_{2}\right) \int_{0}^{L} \varphi_{t}(x, t) \psi_{x t}(x, t) d x \\
& -\int_{0}^{L}\left(\varphi_{x}(x, t)+\psi(x, t)\right) \int_{0}^{+\infty} f(s) \psi_{t}(x, t-s) d s d x \\
& +\left(k_{2} \psi_{x}(L, t)-\int_{0}^{+\infty} g(s) \psi_{x}(L, t-s) d s\right) \varphi_{x}(L, t) \\
& -\left(k_{2} \psi_{x}(0, t)-\int_{0}^{+\infty} g(s) \psi_{x}(0, t-s) d s\right) \varphi_{x}(0, t)+\frac{\rho_{1}}{k_{1}} \int_{0}^{L} \varphi_{t}(x, t) \int_{0}^{+\infty} g^{\prime}(s) \eta_{x}(x, t, s) d s d x .
\end{aligned}
$$

By using (3.33) and Young's inequality for the last three terms in this equality, we get (3.38).

To handle the boundary terms in (3.38), we proceed as in Ref. 2.

Lemma 3.6. Let $m(x):=2-\frac{4}{L} x$. The functionals

$$
J_{1}(t):=\rho_{2} \int_{0}^{L} m(x) \psi_{t}(x, t)\left(k_{2} \psi_{x}(x, t)-\int_{0}^{+\infty} g(s) \psi_{x}(x, t-s) d s\right) d x
$$

and

$$
J_{2}(t):=\rho_{1} \int_{0}^{L} m(x) \varphi_{t}(x, t) \varphi_{x}(x, t) d x
$$

satisfy, for all $\varepsilon>0$ and for some $c_{2}, c_{3}>0$ (not depending neither on f nor on $\varepsilon$ ),

$$
\begin{aligned}
J_{1}^{\prime}(t) \leq & c_{2}\left(1+\frac{1}{\varepsilon}\right) \int_{0}^{L} \psi_{x}^{2}(x, t) d x-\left(k_{2} \psi_{x}(L, t)-\int_{0}^{+\infty} g(s) \psi_{x}(L, t-s) d s\right)^{2} \\
& -\left(k_{2} \psi_{x}(0, t)-\int_{0}^{+\infty} g(s) \psi_{x}(0, t-s) d s\right)^{2}+\varepsilon k_{1} \int_{0}^{L}\left(\varphi_{x}(x, t)+\psi(x, t)\right)^{2} d x \\
& +c_{\varepsilon} \int_{0}^{L} \int_{0}^{+\infty} g(s) \eta_{x}^{2}(x, t, s) d s d x-c_{2} \int_{0}^{L} \int_{0}^{+\infty} g^{\prime}(s) \eta_{x}^{2}(x, t, s) d s d x \\
& +c_{2} \int_{0}^{L} \psi_{t}^{2}(x, t) d x-k_{2} \int_{0}^{L} m(x) \psi_{x}(x, t) \int_{0}^{+\infty} f(s) \psi_{t}(x, t-s) d s d x \\
& +\int_{0}^{L} m(x)\left(\int_{0}^{+\infty} g(s) \psi_{x}(x, t-s) d s\right) \int_{0}^{+\infty} f(s) \psi_{t}(x, t-s) d s d x
\end{aligned}
$$


and

$$
\begin{aligned}
J_{2}^{\prime}(t) \leq & -k_{1}\left(\varphi_{x}^{2}(L, t)+\varphi_{x}^{2}(0, t)\right) \\
& +c_{3} \int_{0}^{L}\left(\varphi_{t}^{2}(x, t)+\left(\varphi_{x}(x, t)+\psi(x, t)\right)^{2}+\psi_{x}^{2}(x, t)\right) d x .
\end{aligned}
$$

Proof. As in Ref. 2 in case $f \equiv 0$, differentiating $J_{1}$, using the second equation in (1.1) and (3.39), and integrating by parts, we find

$$
\begin{aligned}
J_{1}^{\prime}(t)= & \int_{0}^{L} m(x)\left(k_{2} \psi_{x x}(x, t)-\int_{0}^{+\infty} g(s) \psi_{x x}(x, t-s) d s\right)\left(k_{2} \psi_{x}(x, t)-\int_{0}^{+\infty} g(s) \psi_{x}(x, t-s) d s\right) d x \\
& -k_{1} \int_{0}^{L} m(x)\left(\varphi_{x}(x, t)+\psi(x, t)\right)\left(k_{2} \psi_{x}(x, t)-\int_{0}^{+\infty} g(s) \psi_{x}(x, t-s) d s\right) d x \\
& -\int_{0}^{L} m(x)\left(\int_{0}^{+\infty} f(s) \psi_{t}(x, t-s) d s\right)\left(k_{2} \psi_{x}(x, t)-\int_{0}^{+\infty} g(s) \psi_{x}(x, t-s) d s\right) d x \\
& +\rho_{2} \int_{0}^{L} m(x) \psi_{t}(x, t)\left(k_{2} \psi_{x t}(x, t)-\int_{0}^{+\infty} g(s) \psi_{x t}(x, t-s) d s\right) d x \\
= & -\left(k_{2} \psi_{x}(L, t)-\int_{0}^{+\infty} g(s) \psi_{x}(L, t-s) d s\right)^{2}-\left(k_{2} \psi_{x}(0, t)-\int_{0}^{+\infty} g(s) \psi_{x}(0, t-s) d s\right)^{2} \\
& -\int_{0}^{L} m(x)\left(\int_{0}^{+\infty} f(s) \psi_{t}(x, t-s) d s\right)\left(k_{2} \psi_{x}(x, t)-\int_{0}^{+\infty} g(s) \psi_{x}(x, t-s) d s\right) d x \\
& +\frac{2 k_{2} \rho_{2}}{L} \int_{0}^{L} \psi_{t}^{2}(x, t) d x+\rho_{2} \int_{0}^{L} m(x) \psi_{t}(x, t) \int_{0}^{\infty} g^{\prime}(s) \eta_{x}(x, t, s) d s d x \\
& +\frac{2}{L} \int_{0}^{L}\left(\left(k_{2}-g_{0}\right) \psi_{x}(x, t)+\int_{0}^{+\infty} g(s) \eta_{x}(x, t, s) d s\right)^{2} d x \\
& -k_{1} \int_{0}^{L} m(x)\left(\varphi_{x}(x, t)+\psi(x, t)\right)\left(\left(k_{2}-g_{0}\right) \psi_{x}(x, t)+\int_{0}^{+\infty} g(s) \eta_{x}(x, t, s) d s\right) d x .
\end{aligned}
$$

Using Young's inequality and the fact that $|m(x)| \leq 2$ on $] 0, L[$, we get

$$
\begin{aligned}
& -k_{1} m(x)\left(\varphi_{x}(x, t)+\psi(x, t)\right)\left(\left(k_{2}-g_{0}\right) \psi_{x}(x, t)+\int_{0}^{+\infty} g(s) \eta_{x}(x, t, s) d s\right) \\
\leq & \varepsilon k_{1}\left(\varphi_{x}(x, t)+\psi(x, t)\right)^{2}+\frac{k_{1}}{\varepsilon}\left(\left(k_{2}-g_{0}\right) \psi_{x}(x, t)+\int_{0}^{+\infty} g(s) \eta_{x}(x, t, s) d s\right)^{2} .
\end{aligned}
$$

Developing the last term in this inequality, inserting it in the previous equality and using Young's inequality and (3.33), we find

$$
\begin{aligned}
J_{1}^{\prime}(t) \leq & c_{2}\left(1+\frac{1}{\varepsilon}\right) \int_{0}^{L} \psi_{x}^{2}(x, t) d x-\left(k_{2} \psi_{x}(L, t)-\int_{0}^{+\infty} g(s) \psi_{x}(L, t-s) d s\right)^{2} \\
& -\left(k_{2} \psi_{x}(0, t)-\int_{0}^{+\infty} g(s) \psi_{x}(0, t-s) d s\right)^{2}+c_{2} \int_{0}^{L} \psi_{t}^{2}(x, t) d x \\
& +\varepsilon k_{1} \int_{0}^{L}\left(\varphi_{x}(x, t)+\psi(x, t)\right)^{2} d x-c_{2} \int_{0}^{L} \int_{0}^{+\infty} g^{\prime}(s) \eta_{x}^{2}(x, t, s) d s d x
\end{aligned}
$$




$$
\begin{aligned}
& +c_{2}\left(1+\frac{1}{\varepsilon}\right) \int_{0}^{L}\left(\int_{0}^{+\infty} g(s) \eta_{x}(x, t, s) d s\right)^{2} d x \\
& +\int_{0}^{L} m(x)\left(\int_{0}^{+\infty} g(s) \psi_{x}(x, t-s) d s\right) \int_{0}^{+\infty} f(s) \psi_{t}(x, t-s) d s d x \\
& -k_{2} \int_{0}^{L} m(x) \psi_{x}(x, t) \int_{0}^{+\infty} f(s) \psi_{t}(x, t-s) d s d x \\
& -2 g_{0}\left(\frac{k_{1}}{\varepsilon}+\frac{2}{L}\right) \int_{0}^{L} \psi_{x}(x, t) \int_{0}^{+\infty} g(s) \eta_{x}(x, t, s) d s d x
\end{aligned}
$$

Applying again Young's inequality to the last term in (3.42), and using (3.32), we get (3.40).

On the other hand, using Poincaré's inequality (2.9) for $\psi$, we get

$$
\int_{0}^{L} \varphi_{x}^{2}(x, t) d x \leq 2 \int_{0}^{L}\left(\varphi_{x}(x, t)+\psi(x, t)\right)^{2} d x+2 c_{0} \int_{0}^{L} \psi_{x}^{2}(x, t) d x
$$

Then, similarly, differentiating $J_{2}$, using the first equation in (1.1), integrating by parts and using Young's inequality and (3.43), we obtain (3.41).

Lemma 3.7. For any $\epsilon \in] 0,1[$, the functional

$$
I_{4}(t):=I_{3}(t)+\frac{1}{2 \varepsilon} J_{1}(t)+\frac{\varepsilon}{2 k_{1}} J_{2}(t)
$$

satisfies, for some $c_{4}>0$ (not depending neither on f nor on $\varepsilon$ ),

$$
\begin{aligned}
I_{4}^{\prime}(t) \leq & -\left(\frac{k_{1}}{2}-\varepsilon c_{4}\right) \int_{0}^{L}\left(\varphi_{x}(x, t)+\psi(x, t)\right)^{2} d x+\varepsilon c_{4} \int_{0}^{L} \varphi_{t}^{2}(x, t) d x \\
& +c_{\varepsilon} \int_{0}^{L} \int_{0}^{+\infty}\left(g(s) \eta_{x}^{2}(x, t, s)-g^{\prime}(s) \eta_{x}^{2}(x, t, s)\right) d s d x \\
& +\frac{c_{4}}{\varepsilon^{2}} \int_{0}^{L} \psi_{x}^{2}(x, t) d x+\int_{0}^{L} J_{3}(x, t) \int_{0}^{+\infty} f(s) \psi_{t}(x, t-s) d s d x \\
& +\frac{c_{4}}{\varepsilon} \int_{0}^{L} \psi_{t}^{2}(x, t) d x+\left(\frac{\rho_{1} k_{2}}{k_{1}}-\rho_{2}\right) \int_{0}^{L} \varphi_{t}(x, t) \psi_{x t}(x, t) d x,
\end{aligned}
$$

where

$$
J_{3}(x, t):=-\varphi_{x}(x, t)-\psi(x, t)-\frac{1}{2 \varepsilon} m(x)\left(k_{2} \psi_{x}(x, t)-\int_{0}^{+\infty} g(s) \psi_{x} x, t-s d s\right) .
$$

Proof. Combining (3.38) and (3.40) and (3.41), we obtain (3.44).

Lemma 3.8. The functional

$$
I_{5}(t):=\frac{1}{8} I_{2}(t)+I_{4}(t)
$$


satisfies, for some $c_{5}>0$ (not depending on $f$ ),

$$
\begin{aligned}
I_{5}^{\prime}(t) \leq & -\frac{k_{1}}{4} \int_{0}^{L}\left(\varphi_{x}(x, t)+\psi(x, t)\right)^{2} d x+c_{5} \int_{0}^{L}\left(\psi_{t}^{2}(x, t)+\psi_{x}^{2}(x, t)\right) d x \\
& +c_{5} \int_{0}^{L} \int_{0}^{\infty}\left(g(s) \eta_{x}^{2}(x, t, s)-g^{\prime}(s) \eta_{x}^{2}(x, t, s)\right) d s d x \\
& -\frac{\rho_{1}}{16} \int_{0}^{L} \varphi_{t}^{2}(x, t) d x+\int_{0}^{L} J_{4}(x, t) \int_{0}^{+\infty} f(s) \psi_{t}(x, t-s) d s d x \\
& +\left(\frac{\rho_{1} k_{2}}{k_{1}}-\rho_{2}\right) \int_{0}^{L} \varphi_{t}(x, t) \psi_{x t}(x, t) d x,
\end{aligned}
$$

where

$$
J_{4}(x, t):=J_{3}(x, t)+\frac{1}{8} \psi(x, t)
$$

Proof. Estimates (3.36) and (3.44) with $0<\varepsilon<\min \left\{\frac{k_{1}}{8 c_{4}}, \frac{\rho_{1}}{16 c_{4}}\right\}$ small enough imply (3.46)

Now, as in Ref. 2, we use a function $w$ to get a crucial estimate.

Lemma 3.9. Let

$$
w(x, t):=-\int_{0}^{x} \psi(y, t) d y+\frac{1}{L}\left(\int_{0}^{L} \psi(y, t) d y\right) x .
$$

Then the functional

$$
I_{6}(t):=\int_{0}^{L}\left(\rho_{1} \varphi_{t}(x, t) w(x, t)+\rho_{2} \psi_{t}(x, t) \psi(x, t)\right) d x
$$

satisfies, for all $\left.\varepsilon_{1} \in\right] 0,1\left[\right.$ and for some $c_{6}>0$ (not depending neither on $f$ nor on $\varepsilon_{1}$ ),

$$
\begin{aligned}
I_{6}^{\prime}(t) \leq & -\frac{k_{2}-g_{0}}{2} \int_{0}^{L} \psi_{x}^{2}(x, t) d x+\frac{c_{6}}{\varepsilon_{1}} \int_{0}^{L} \psi_{t}^{2}(x, t) d x \\
& +\varepsilon_{1} \int_{0}^{L} \varphi_{t}^{2}(x, t) d x+c_{6} \int_{0}^{L} \int_{0}^{+\infty} g(s) \eta_{x}^{2}(x, t, s) d s d x \\
& -\int_{0}^{L} \psi(x, t) \int_{0}^{+\infty} f(s) \psi_{t}(x, t-s) d s d x .
\end{aligned}
$$

Proof. The fact that $-w_{x x}=\psi_{x}$ and $w(0, t)=w(L, t)=0$ imply that

$$
\begin{aligned}
\int_{0}^{L} w_{x}^{2}(x, t) d x= & \int_{0}^{L} \psi_{x}(x, t) w(x, t) d x=-\int_{0}^{L} \psi(x, t) w_{x}(x, t) d x \\
& \leq\left(\int_{0}^{L} \psi^{2}(x, t) d x\right)^{\frac{1}{2}}\left(\int_{0}^{L} w_{x}^{2}(x, t) d x\right)^{\frac{1}{2}},
\end{aligned}
$$

which gives, using (2.9) for $w_{t}$ (note that $w_{t}(0, t)=w_{t}(L, t)=0$ ),

$$
\int_{0}^{L} w_{x}^{2}(x, t) d x \leq \int_{0}^{L} \psi^{2}(x, t) d x \quad \text { and } \quad \int_{0}^{L} w_{t}^{2}(x, t) d x \leq c_{0} \int_{0}^{L} \psi_{t}^{2}(x, t) d x .
$$

On the other hand,

$$
-k_{1} \int_{0}^{L}\left(\varphi_{x}(x, t)+\psi(x, t)\right)\left(w_{x}(x, t)+\psi(x, t)\right) d x
$$




$$
\begin{aligned}
& =-k_{1}\left(\int_{0}^{L}\left(\varphi_{x}(x, t)+\psi(x, t)\right) d x\right)\left(\frac{1}{L} \int_{0}^{L} \psi(y, t) d y\right) \\
& =\frac{-k_{1}}{L}\left(\int_{0}^{L} \psi(x, t) d x\right)^{2} \\
& \leq 0 .
\end{aligned}
$$

Therefore, as in Ref. 2 in case $f \equiv 0$, by differentiating $I_{6}$, using the first two equations in (1.1), integrating by parts and using (3.49) and (3.50) and Young's inequality for $\varphi_{t} w_{t}$, we find

$$
\begin{aligned}
I_{6}^{\prime}(t) \leq & -\left(k_{2}-g_{0}\right) \int_{0}^{L} \psi_{x}^{2}(x, t) d x+\frac{c_{6}}{\varepsilon_{1}} \int_{0}^{L} \psi_{t}^{2}(x, t) d x \\
& +\varepsilon_{1} \int_{0}^{L} \varphi_{t}^{2}(x, t) d x-\int_{0}^{L} \psi(x, t) \int_{0}^{+\infty} f(s) \psi_{t}(x, t-s) d s d x \\
& -\int_{0}^{L} \psi_{x}(x, t) \int_{0}^{+\infty} g(s) \eta_{x}(x, t, s) d s d x .
\end{aligned}
$$

The use of Young's inequality and (3.32) for the last term in (3.51) gives (3.48).

Now, we introduce a new functional $I_{7}$ which plays a crucial role in dealing with the distributed time delay.

Lemma 3.10. Let

$$
I_{7}(t):=e^{-\hat{\gamma}(t)} \int_{0}^{L} \int_{0}^{+\infty} e^{\tilde{\gamma}(s)}|f(s)|\left(\int_{t-s}^{t} e^{\hat{\gamma}(\tau)} \psi_{t}^{2}(x, \tau) d \tau\right) d s d x
$$

where $\tilde{\gamma}$ and $\gamma$ are defined in assumption (A4), and

$$
\hat{\gamma}(t):=\int_{0}^{t} \gamma(s) d s
$$

The functional $I_{7}$ satisfies

$$
I_{7}^{\prime}(t) \leq g(0) \beta_{0} \int_{0}^{L} \psi_{t}^{2}(x, t) d x-\gamma(t) I_{7}(t)-\int_{0}^{L} \int_{0}^{+\infty}|f(s)| \psi_{t}^{2}(x, t-s) d s d x
$$

where $\beta_{0}$ is defined in (3.4).

Proof. First, thanks to (2.9), (2.14), and (3.5) we have (note also that $g$ is nonincreasing and $\hat{\gamma}$ is increasing)

$$
\begin{aligned}
I_{7}(t) & \leq \int_{0}^{L} \int_{0}^{+\infty} \beta(s) g(s)\left(\int_{t-s}^{t} \psi_{t}^{2}(x, \tau) d \tau\right) d s d x \\
& \leq \int_{0}^{L} \int_{0}^{+\infty} \beta(s)\left(\int_{t-s}^{t} g(t-\tau) \psi_{t}^{2}(x, \tau) d \tau\right) d s d x \\
& \leq c_{0} \int_{0}^{L} \int_{0}^{+\infty} \beta(s)\left(\int_{0}^{s} g(\tau) \psi_{x t}^{2}(x, t-\tau) d \tau\right) d s d x \\
& \leq c_{0} \int_{0}^{L} \int_{0}^{+\infty} \beta(s)\left(\int_{0}^{+\infty} g(\tau) \eta_{x s}^{2}(x, t, \tau) d \tau\right) d s d x
\end{aligned}
$$

thus, due to (3.4) and the fact that $\eta_{s} \in L_{g}^{2}\left(\mathbb{R}_{+}, H_{0}^{1}(] 0, L[)\right)$ (in virtue of (2.16) with $n=1$ ),

$$
I_{7}(t) \leq c_{0} \beta_{0}\left\|\eta_{s}(x, t, \cdot)\right\|_{L_{g}^{2}\left(\mathbb{R}_{+}, H_{0}^{1}(j 0, L[))\right.}^{2},
$$


and therefore, the functional $I_{7}$ is well-defined. Moreover, using again (3.5), a simple and direct differentiation gives

$$
\begin{aligned}
I_{7}^{\prime}(t)= & -\gamma(t) I_{7}(t)+\left(\int_{0}^{+\infty} e^{\tilde{\gamma}(s)}|f(s)| d s\right) \int_{0}^{L} \psi_{t}^{2}(x, t) d x \\
& \quad-\int_{0}^{L} \int_{0}^{+\infty} e^{\tilde{\gamma}(s)+\hat{\gamma}(t-s)-\hat{\gamma}(t)}|f(s)| \psi_{t}^{2}(x, t-s) d s d x \\
\leq & -\gamma(t) I_{7}(t)+\left(\int_{0}^{+\infty} \beta(s) g(s) d s\right) \int_{0}^{L} \psi_{t}^{2}(x, t) d x \\
& \quad-\int_{0}^{L} \int_{0}^{+\infty} e^{\tilde{\gamma}(s)+\hat{\gamma}(t-s)-\hat{\gamma}(t)}|f(s)| \psi_{t}^{2}(x, t-s) d s d x \\
\leq- & \gamma(t) I_{7}(t)+g(0) \beta_{0} \int_{0}^{L} \psi_{t}^{2}(x, t) d x \\
& \quad-\int_{0}^{L} \int_{0}^{+\infty} e^{\tilde{\gamma}(s)+\hat{\gamma}(t-s)-\hat{\gamma}(t)}|f(s)| \psi_{t}^{2}(x, t-s) d s d x .
\end{aligned}
$$

On the other hand, the function $h(t):=\tilde{\gamma}(s)+\hat{\gamma}(t-s)-\hat{\gamma}(t)$, for $s \geq 0$ fixed and $t \geq 0$, satisfies $h^{\prime}(t)=\gamma(t-s)-\gamma(t)$. Then $h$ is nondecreasing, for $t \geq \frac{s}{2}$, and it is nonincreasing, for $t \leq \frac{s}{2}$, because $\gamma$ is even and nonincreasing on $\mathbb{R}_{+}$. Therefore, $h(t) \geq h\left(\frac{s}{2}\right)=0$, and (3.54) follows at once.

Now, let $N, N_{1}$, and $N_{2}$ be positive constants (which will be fixed latter on). We define the functional

$$
\mathscr{L}_{1}(t):=N E(t)+N_{1} I_{1}(t)+I_{5}(t)+N_{2} I_{6}(t)+I_{7}(t) .
$$

At this step, we distinguish three cases depending on (1.2) and (3.2)-(3.3).

\section{Equal speed propagation and exponential decay of g: (1.2) and (3.2) hold}

By combining (3.31), (3.34), (3.46), (3.48), and (3.54) taking $\delta=\frac{k_{1}}{8 N_{1}}$ and using (3.2), we get

$$
\mathscr{L}_{1}^{\prime}(t)
$$

$$
\begin{aligned}
\leq & -\left(\frac{\left(k_{2}-g_{0}\right) N_{2}}{2}-c_{5}-\frac{k_{1}}{8}\right) \int_{0}^{L} \psi_{x}^{2}(x, t) d x-\left(\frac{\rho_{1}}{16}-\varepsilon_{1} N_{2}\right) \int_{0}^{L} \varphi_{t}^{2}(x, t) d x \\
& -\frac{k_{1}}{8} \int_{0}^{L}\left(\varphi_{x}(x, t)+\psi(x, t)\right)^{2} d x-\int_{0}^{L} \int_{0}^{+\infty} g(s) \eta_{x}^{2}(x, t, s) d s d x \\
& -\left(\rho_{2}\left(g_{0} N_{1}-\frac{k_{1}}{8}\right)-\frac{c_{6} N_{2}}{\varepsilon_{1}}-g(0) \beta_{0}-c_{5}\right) \int_{0}^{L} \psi_{t}^{2}(x, t) d x-\gamma(t) I_{7}(t) \\
& +\left(\frac{N}{2}-c_{N_{1}, N_{2}}\right) \int_{0}^{L} \int_{0}^{+\infty} g^{\prime}(s) \eta_{x}^{2}(x, t, s) d s d x-\int_{0}^{L} \int_{0}^{+\infty}|f(s)| \psi_{t}^{2}(x, t-s) d s d x \\
& +\int_{0}^{L} J_{5}(x, t) \int_{0}^{+\infty} f(s) \psi_{t}(x, t-s) d s d x+\left(\frac{\rho_{1} k_{2}}{k_{1}}-\rho_{2}\right) \int_{0}^{L} \varphi_{t}(x, t) \psi_{x t}(x, t) d x,
\end{aligned}
$$

where

$$
J_{5}(x, t):=J_{4}(x, t)-N \psi_{t}(x, t)-N_{2} \psi(x, t)+N_{1} \int_{0}^{+\infty} g(s) \eta(x, t, s) d s
$$


Due to (1.2), the last term in (3.58) vanishes. Now, we choose $N_{2}$ large enough such that

$$
\frac{\left(k_{2}-g_{0}\right) N_{2}}{2}-c_{5}-\frac{k_{1}}{8}>0
$$

$\left(N_{2}\right.$ exists according to (2.1)), then we take $\left.\varepsilon_{1} \in\right] 0,1[$ small enough so that

$$
\frac{\rho_{1}}{16}-\varepsilon_{1} N_{2}>0
$$

Next, we pick $N_{1}$ so large such that

$$
\rho_{2}\left(g_{0} N_{1}-\frac{k_{1}}{8}\right)-\frac{c_{6} N_{2}}{\varepsilon_{1}}-g(0) \beta_{0}-c_{5}>0
$$

$\left(N_{1}\right.$ exists thanks to (3.1)). On the other hand, by the definition of the functionals $E, I_{1}-I_{6}$, and $J_{1}-J_{2}$, and the use of (2.15), (2.9) and Young's inequality, there exists a positive constant $c_{7}$ (not depending neither on $f$ nor on $N$ ) such that

$$
\left|N_{1} I_{1}(t)+I_{5}(t)+N_{2} I_{6}(t)\right| \leq c_{7} E(t), \quad \forall t \in \mathbb{R}_{+},
$$

which implies that

$$
\left(N-c_{7}\right) E(t) \leq \mathscr{L}_{1}(t)-I_{7}(t) \leq\left(N+c_{7}\right) E(t), \quad \forall t \in \mathbb{R}_{+} .
$$

Then, we choose $N$ large enough such that

$$
N>\max \left\{c_{7}, 2 c_{N_{1}, N_{2}}\right\},
$$

so $E \sim \mathscr{L}_{1}-I_{7}$ holds and, from (3.58) and the definition of $E$, we obtain, for some $c_{8}>0$ (not depending on $f$ ),

$$
\begin{aligned}
\mathscr{L}_{1}^{\prime}(t) \leq & -c_{8} E(t)-\int_{0}^{L} \int_{0}^{+\infty}|f(s)| \psi_{t}^{2}(x, t-s) d s d x \\
& -\gamma(t) I_{7}(t)+\int_{0}^{L} J_{5}(x, t) \int_{0}^{+\infty} f(s) \psi_{t}(x, t-s) d s d x .
\end{aligned}
$$

Similar to (3.61), from the definition of $E$ and $J_{3}-J_{5}$, and using (2.9) and Young's inequality, we find that there exists a positive constant $c_{9}$ (not depending on $f$ ) such that

$$
\int_{0}^{L} J_{5}^{2}(x, t) d x \leq c_{9} E(t) .
$$

Therefore, applying Cauchy-Schwarz and Young's inequalities, we get, for

$$
\epsilon^{\prime}=2\left(\int_{0}^{+\infty}|f(s)| d s\right)^{-1}
$$

(if $\int_{0}^{+\infty}|f(s)| d s=0$, then $f \equiv 0$, and therefore, the two terms in (3.64) depending on $f$ vanish),

$$
\begin{gathered}
\int_{0}^{L} J_{5}(x, t) \int_{0}^{+\infty} f(s) \psi_{t}(x, t-s) d s d x \\
\leq\left(\int_{0}^{L}\left(\int_{0}^{+\infty} f(s) \psi_{t}(x, t-s) d s\right)^{2} d x\right)^{\frac{1}{2}}\left(\int_{0}^{L} J_{5}^{2}(x, t) d x\right)^{\frac{1}{2}} \\
\leq \frac{\epsilon^{\prime}}{2}\left(\int_{0}^{+\infty}|f(s)| d s\right) \int_{0}^{L} \int_{0}^{+\infty}|f(s)| \psi_{t}^{2}(x, t-s) d s d x+\frac{1}{2 \epsilon^{\prime}} \int_{0}^{L} J_{5}^{2}(x, t) d x \\
\leq \int_{0}^{L} \int_{0}^{+\infty}|f(s)| \psi_{t}^{2}(x, t-s) d s d x+\frac{c_{9}}{4}\left(\int_{0}^{+\infty}|f(s)| d s\right) E(t) .
\end{gathered}
$$


Hence, under condition (3.8) with

$$
\delta_{0}=\frac{4 c_{8}}{c_{9}}
$$

(noting that $\delta_{0}$ is positive and does not depend on $f$ ) and by combining (3.64) and (3.66), we find, for some $c_{10}>0$,

$$
\mathscr{L}_{1}^{\prime}(t) \leq-c_{10} E(t)-\gamma(t) I_{7}(t), \quad \forall t \in \mathbb{R}_{+} .
$$

By combining (3.62) and (3.68), we obtain

$$
\mathscr{L}_{1}^{\prime}(t) \leq-\delta_{1} \min \{1, \gamma(t)\} \mathscr{L}(t), \quad \forall t \in \mathbb{R}_{+},
$$

where $\delta_{1}=\min \left\{\frac{c_{10}}{N+c_{7}}, 1\right\}$. Then, an integration of the differential inequality (3.69) over [0,t] gives

$$
\mathscr{L}_{1}(t) \leq \mathscr{L}_{1}(0) e^{-\delta_{1} \phi(t)}, \quad \forall t \in \mathbb{R}_{+},
$$

where $\phi$ is defined in (3.10). Consequently, the choice (3.63) of $N$ and the relations (3.30)(3.62)(3.70) lead to

$$
\|\mathscr{U}(t)\|_{\mathscr{H}}^{2}=2 E(t) \leq \frac{2}{N-c_{7}} \mathscr{L}_{1}(t) \leq \frac{2 \mathscr{L}_{1}(0)}{N-c_{7}} e^{-\delta_{1} \phi(t)}, \quad \forall t \in \mathbb{R}_{+},
$$

which is the decay estimate (3.9) with $\delta_{2}=\frac{2 \mathscr{L}_{1}(0)}{N-c_{7}}$.

\section{Nonequal speed propagation and exponential decay of $g:(1.2)$ does not hold and (3.2) holds}

To deal with the last term in (3.58) (which can not be absorbed by $E$ ) and get (3.11), we appeal to some ideas of Refs. 1, 8, and 13 based on the energies of high orders defined by

$$
E_{k}(t)=\frac{1}{2}\left\|\mathscr{U}^{(k)}(t)\right\|_{\mathscr{H}}^{2}, \quad \forall \mathscr{U}_{0} \in \mathscr{D}\left(\mathscr{A}^{k}\right), \quad k=0, \cdots, n
$$

(so $E_{0}=E$ ). As for (3.31), $E_{k}$ satisfies

$$
\begin{aligned}
E_{k}^{\prime}(t)= & \frac{1}{2} \int_{0}^{L} \int_{0}^{+\infty} g^{\prime}(s)\left(\partial_{t}^{k} \eta_{x}\right)^{2}(x, t, s) d s d x \\
& -\int_{0}^{L} \partial_{t}^{k+1} \psi(x, t) \int_{0}^{+\infty} f(s) \partial_{t}^{k+1} \psi(x, t-s) d s d x
\end{aligned}
$$

We start by proving two lemmas, where the first one is given in Ref. 8, while the second one is introduced in the present paper to cope with some delay terms.

Lemma 3.11. For any $\varepsilon>0$, we have

$$
\begin{gathered}
\left(\frac{\rho_{1} k_{2}}{k_{1}}-\rho_{2}\right) \int_{0}^{L} \varphi_{t}(x, t) \psi_{x t}(x, t) d x \\
\leq \epsilon\left|\frac{\rho_{1} k_{2}}{k_{1}}-\rho_{2}\right| \int_{0}^{L} \varphi_{t}^{2}(x, t) d x-\frac{1}{\epsilon \theta_{1} g_{0}}\left|\frac{\rho_{1} k_{2}}{k_{1}}-\rho_{2}\right| E_{1}^{\prime}(t) \\
-\frac{g(0)}{2 \epsilon g_{0}^{2}}\left|\frac{\rho_{1} k_{2}}{k_{1}}-\rho_{2}\right| \int_{0}^{L} \int_{0}^{+\infty} g^{\prime}(s) \eta_{x}^{2}(x, t, s) d s d x \\
-\frac{1}{\epsilon \theta_{1} g_{0}}\left|\frac{\rho_{1} k_{2}}{k_{1}}-\rho_{2}\right| \int_{0}^{L} \psi_{t t}(x, t) \int_{0}^{+\infty} f(s) \psi_{t t}(x, t-s) d s d x .
\end{gathered}
$$


Proof. We proceed as in Ref. 8. By recalling that $g_{0}=\int_{0}^{+\infty} g(s) d s$, we have

$$
\begin{gathered}
\left(\frac{\rho_{1} k_{2}}{k_{1}}-\rho_{2}\right) \int_{0}^{L} \varphi_{t}(x, t) \psi_{x t}(x, t) d x \\
=\frac{1}{g_{0}}\left(\frac{\rho_{1} k_{2}}{k_{1}}-\rho_{2}\right) \int_{0}^{L} \varphi_{t}(x, t) \int_{0}^{+\infty} g(s) \eta_{x t}(x, t, s) d s d x \\
+\frac{1}{g_{0}}\left(\frac{\rho_{1} k_{2}}{k_{1}}-\rho_{2}\right) \int_{0}^{L} \varphi_{t}(x, t) \int_{0}^{+\infty} g(s) \psi_{x t}(x, t-s) d s d x .
\end{gathered}
$$

Using Young's inequality and (3.32) (for $v=\eta_{x t}$ ), we get, for all $\varepsilon>0$,

$$
\begin{gathered}
\frac{1}{g_{0}}\left(\frac{\rho_{1} k_{2}}{k_{1}}-\rho_{2}\right) \int_{0}^{L} \varphi_{t}(x, t) \int_{0}^{+\infty} g(s) \eta_{x t}(x, t, s) d s d x \\
\leq \frac{\epsilon}{2}\left|\frac{\rho_{1} k_{2}}{k_{1}}-\rho_{2}\right| \int_{0}^{L} \varphi_{t}^{2}(x, t) d x+\frac{1}{2 \epsilon g_{0}}\left|\frac{\rho_{1} k_{2}}{k_{1}}-\rho_{2}\right| \int_{0}^{L} \int_{0}^{+\infty} g(s) \eta_{x t}^{2}(x, t, s) d s d x .
\end{gathered}
$$

Moreover, using (3.2) and (3.72) for $k=1$, we get

$$
\begin{gathered}
\frac{1}{2 \epsilon g_{0}}\left|\frac{\rho_{1} k_{2}}{k_{1}}-\rho_{2}\right| \int_{0}^{L} \int_{0}^{+\infty} g(s) \eta_{x t}^{2}(x, t, s) d s d x \\
\leq-\frac{1}{\epsilon \theta_{1} g_{0}}\left|\frac{\rho_{1} k_{2}}{k_{1}}-\rho_{2}\right| E_{1}^{\prime}(t) \\
-\frac{1}{\epsilon \theta_{1} g_{0}}\left|\frac{\rho_{1} k_{2}}{k_{1}}-\rho_{2}\right| \int_{0}^{L} \psi_{t t}(x, t) \int_{0}^{+\infty} f(s) \psi_{t t}(x, t-s) d s d x .
\end{gathered}
$$

On the other hand, by integrating by parts with respect to $s$ and using (3.39) and (3.33) (for $v=\eta_{x}$ ) and Young's inequality, we have, for all $\varepsilon>0$ (note also that $\eta_{x}(x, t, 0)=\lim _{s \rightarrow+\infty} g(s) \eta_{x}(x, t, s)$ $=0$ due to (2.13) and (2.16) for $n \geq 1)$,

$$
\begin{gathered}
\quad \frac{1}{g_{0}}\left(\frac{\rho_{1} k_{2}}{k_{1}}-\rho_{2}\right) \int_{0}^{L} \varphi_{t}(x, t) \int_{0}^{+\infty} g(s) \psi_{x t}(x, t-s) d s d x \\
=\frac{1}{g_{0}}\left(\frac{\rho_{1} k_{2}}{k_{1}}-\rho_{2}\right) \int_{0}^{L} \varphi_{t}(x, t) \int_{0}^{+\infty} g(s) \eta_{x s}(x, t, s) d s d x \\
=\frac{1}{g_{0}}\left(\frac{\rho_{1} k_{2}}{k_{1}}-\rho_{2}\right) \int_{0}^{L} \varphi_{t}(x, t) \int_{0}^{+\infty}\left(-g^{\prime}(s)\right) \eta_{x}(x, t, s) d s d x \\
\leq \frac{\epsilon}{2}\left|\frac{\rho_{1} k_{2}}{k_{1}}-\rho_{2}\right| \int_{0}^{L} \varphi_{t}^{2}(x, t) d x-\frac{g(0)}{2 \epsilon g_{0}^{2}}\left|\frac{\rho_{1} k_{2}}{k_{1}}-\rho_{2}\right| \int_{0}^{L} \int_{0}^{+\infty} g^{\prime}(s) \eta_{x}^{2}(x, t, s) d s d x .
\end{gathered}
$$

Inserting (3.75)-(3.77) into (3.74), we find (3.73).

Lemma 3.12. The functional

$$
I_{8}(t):=\int_{0}^{L} \psi_{t}(x, t)\left(\frac{f(0)}{2} \psi_{t}(x, t)-\int_{0}^{+\infty} f^{\prime \prime}(s) \eta(x, t, s) d s\right) d x
$$

satisfies ( $\tilde{\alpha}$ is defined in (3.7)),

$$
\begin{aligned}
I_{8}^{\prime}(t) \leq & \int_{0}^{L} \psi_{t t}(x, t) \int_{0}^{+\infty} f(s) \psi_{t t}(x, t-s) d s d x+\left(\alpha g(0)+\frac{1}{2}\right) \int_{0}^{L} \psi_{t}^{2}(x, t) d s d x \\
& -\frac{\tilde{\alpha}^{2} g_{0} c_{0}}{2 \theta_{1}} \int_{0}^{L} \int_{0}^{+\infty} g^{\prime}(s) \eta_{x}^{2}(x, t, s) d s d x .
\end{aligned}
$$


Proof. From (2.14), we conclude that $\eta_{s s}(x, t, s)=-\psi_{t t}(x, t-s)$ (recall that $\eta_{s s} \in$ $L_{g}^{2}\left(\mathbb{R}_{+}, H_{0}^{1}(] 0, L[)\right)$, for any $t \in \mathbb{R}_{+}$fixed, since $\mathscr{U}_{0} \in \mathscr{D}\left(\mathscr{A}^{n}\right)$ with $\left.n \geq 2\right)$, and then

$$
\int_{0}^{L} \psi_{t t}(x, t) \int_{0}^{+\infty} f(s) \psi_{t t}(x, t-s) d s d x=-\int_{0}^{L} \psi_{t t}(x, t) \int_{0}^{+\infty} f(s) \eta_{s s}(x, t, s) d s d x .
$$

By integrating by parts with respect to $s$ and using the fact that $\eta_{s}(x, t, 0)=\psi_{t}(x, t)$ (du to (2.14)), $\eta(x, t, 0)=0$ (thanks to (2.13)) and $\lim _{s \rightarrow+\infty} f(s) \eta_{s}(x, t, s)=\lim _{s \rightarrow+\infty} f^{\prime}(s) \eta(x, t, s)=0$ (according to (2.3) and (2.16) with $n \geq 2$ ), we get

$$
\begin{aligned}
\int_{0}^{L} \psi_{t t}(x, t) \int_{0}^{+\infty} f(s) \psi_{t t}(x, t-s) d s d x & =\int_{0}^{L} \psi_{t t}(x, t)\left(f(0) \psi_{t}(x, t)-\int_{0}^{+\infty} f^{\prime \prime}(s) \eta(x, t, s) d s\right) d x \\
& =I_{8}^{\prime}(t)+\int_{0}^{L} \psi_{t}(x, t) \int_{0}^{+\infty} f^{\prime \prime}(s) \eta_{t}(x, t, s) d s d x .
\end{aligned}
$$

Again, using $\eta(x, t, 0)=\lim _{s \rightarrow+\infty} f^{\prime}(s)=\lim _{s \rightarrow+\infty} f^{\prime}(s) \eta(x, t, s)=0$ (in virtue of (2.3), (2.13), (3.7), and (2.16) with $n \geq 1$ ), and integrating by parts with respect to $s$, we find

$$
\begin{aligned}
I_{8}^{\prime}(t)= & \int_{0}^{L} \psi_{t t}(x, t) \int_{0}^{+\infty} f(s) \psi_{t t}(x, t-s) d s d x \\
& -\int_{0}^{L} \psi_{t}(x, t) \int_{0}^{+\infty} f^{\prime \prime}(s)\left(\psi_{t}(x, t)-\eta_{s}(x, t, s)\right) d s d x \\
= & \int_{0}^{L} \psi_{t t}(x, t) \int_{0}^{+\infty} f(s) \psi_{t t}(x, t-s) d s d x+f^{\prime}(0) \int_{0}^{L} \psi_{t}^{2}(x, t) d x \\
& -\int_{0}^{L} \psi_{t}(x, t) \int_{0}^{+\infty} f^{\prime \prime \prime}(s) \eta(x, t, s) d s d x .
\end{aligned}
$$

On the other hand, using (2.3), we have

$$
f^{\prime}(0) \int_{0}^{L} \psi_{t}^{2}(x, t) d x \leq \alpha g(0) \int_{0}^{L} \psi_{t}^{2}(x, t) d x .
$$

Moreover, using (3.7), Young's inequality, (3.32) (for $v=|\eta|$ ) and (2.9), we obtain

$$
\begin{aligned}
& -\int_{0}^{L} \psi_{t}(x, t) \int_{0}^{+\infty} f^{\prime \prime \prime}(s) \eta(x, t, s) d s d x \\
& \quad \leq \frac{1}{2} \int_{0}^{L} \psi_{t}^{2}(x, t) d x+\frac{\tilde{\alpha}^{2} g_{0} c_{0}}{2} \int_{0}^{L} \int_{0}^{+\infty} g(s) \eta_{x}^{2}(x, t, s) d s d x .
\end{aligned}
$$

Inserting these two inequalities in the previous equality, we arrive at

$$
\begin{aligned}
I_{8}^{\prime}(t) \leq & \int_{0}^{L} \psi_{t t}(x, t) \int_{0}^{+\infty} f(s) \psi_{t t}(x, t-s) d s d x+\left(\alpha g(0)+\frac{1}{2}\right) \int_{0}^{L} \psi_{t}^{2}(x, t) d s d x \\
& +\frac{\tilde{\alpha}^{2} g_{0} c_{0}}{2} \int_{0}^{L} \int_{0}^{+\infty} g(s) \eta_{x}^{2}(x, t, s) d s d x .
\end{aligned}
$$

Applying (3.2) to the last term of (3.80) gives (3.79).

Now, let us consider the functional

$$
\mathscr{L}_{2}(t):=\mathscr{L}_{1}(t)+\frac{1}{\epsilon g_{0} \theta_{1}}\left|\frac{\rho_{1} k_{2}}{k_{1}}-\rho_{2}\right|\left(E_{1}(t)+I_{8}(t)\right) .
$$

By combining (3.58), (3.73), and (3.79) we get

$$
\mathscr{L}_{2}^{\prime}(t)
$$




$$
\begin{aligned}
\leq & -\left(\frac{\left(k_{2}-g_{0}\right) N_{2}}{2}-c_{5}-\frac{k_{1}}{8}\right) \int_{0}^{L} \psi_{x}^{2}(x, t) d x-\left(\frac{\rho_{1}}{16}-\varepsilon_{1} N_{2}-\epsilon\left|\frac{\rho_{1} k_{2}}{k_{1}}-\rho_{2}\right|\right) \int_{0}^{L} \varphi_{t}^{2}(x, t) d x \\
& -\frac{k_{1}}{8} \int_{0}^{L}\left(\varphi_{x}(x, t)+\psi(x, t)\right)^{2} d x-\int_{0}^{L} \int_{0}^{+\infty} g(s) \eta_{x}^{2}(x, t, s) d s d x \\
& -\left(\rho_{2}\left(g_{0} N_{1}-\frac{k_{1}}{8}\right)-\frac{c_{6} N_{2}}{\varepsilon_{1}}-\frac{1}{\epsilon g_{0} \theta_{1}}\left(\alpha g(0)+\frac{1}{2}\right)\left|\frac{\rho_{1} k_{2}}{k_{1}}-\rho_{2}\right|-g(0) \beta_{0}-c_{5}\right) \int_{0}^{L} \psi_{t}^{2}(x, t) d x \\
& +\left(\frac{N}{2}-\left(\frac{g(0)}{2 \epsilon g_{0}^{2}}+\frac{\tilde{\alpha}^{2} c_{0}}{2 \epsilon \theta_{1}^{2}}\right)\left|\frac{\rho_{1} k_{2}}{k_{1}}-\rho_{2}\right|-c_{N_{1}, N_{2}}\right) \int_{0}^{L} \int_{0}^{+\infty} g^{\prime}(s) \eta_{x}^{2}(x, t, s) d s d x-\gamma(t) I_{7}(t) \\
& -\int_{0}^{L} \int_{0}^{+\infty}|f(s)| \psi_{t}^{2}(x, t-s) d s d x+\int_{0}^{L} J_{5}(x, t) \int_{0}^{+\infty} f(s) \psi_{t}(x, t-s) d s d x .
\end{aligned}
$$

First, we choose $N_{2}$ as in (3.60), and then we take $\varepsilon=\varepsilon_{1}$ and $\left.\varepsilon_{1} \in\right] 0,1[$ small enough so that

$$
\frac{\rho_{1}}{16}-\epsilon_{1}\left(N_{2}+\left|\frac{\rho_{1} k_{2}}{k_{1}}-\rho_{2}\right|\right)>0 .
$$

Next, we pick $N_{1}$ so large such that

$$
\rho_{2}\left(g_{0} N_{1}-\frac{k_{1}}{8}\right)-\frac{1}{\varepsilon_{1}}\left(c_{6} N_{2}+\frac{1}{g_{0} \theta_{1}}\left(\alpha g(0)+\frac{1}{2}\right)\left|\frac{\rho_{1} k_{2}}{k_{1}}-\rho_{2}\right|\right)-g(0) \beta_{0}-c_{5}>0
$$

$\left(N_{1}\right.$ exists due to (3.1)). On the other hand, using (2.3) and (3.7), Young's inequality, (3.32) (for $v=|\eta|)$ and (2.9), we find

$$
\left|I_{8}(t)\right| \leq \frac{1+\alpha g(0)}{2} \int_{0}^{L} \psi_{t}^{2}(x, t) d x+\frac{\tilde{\alpha}^{2} g_{0} c_{0}}{2} \int_{0}^{L} \int_{0}^{+\infty} g(s) \eta_{x}^{2}(x, t, s) d s d x .
$$

Consequently, in virtue of (2.15) and (3.30), there exists a positive constant $\tilde{c}_{7}$ (not depending neither on $\int_{0}^{+\infty}|f(s)| d s$ nor on $N$ ) such that

$$
\frac{1}{\epsilon g_{0} \theta_{1}}\left|\frac{\rho_{1} k_{2}}{k_{1}}-\rho_{2}\right|\left|I_{8}(t)\right| \leq \tilde{c}_{7} E(t), \quad \forall t \in \mathbb{R}_{+},
$$

which implies that, for all $t \in \mathbb{R}_{+}$, using (3.62) and (3.81),

$$
\left(N-c_{7}-\tilde{c}_{7}\right) E(t) \leq \mathscr{L}_{2}(t)-\frac{1}{\epsilon g_{0} \theta_{1}}\left|\frac{\rho_{1} k_{2}}{k_{1}}-\rho_{2}\right| E_{1}(t)-I_{7}(t) \leq\left(N+c_{7}+\tilde{c}_{7}\right) E(t) .
$$

Finally, we choose $N$ large enough such that

$$
N>\max \left\{c_{7}+\tilde{c}_{7}, 2 c_{N_{1}, N_{2}}+\left(\frac{g(0)}{\epsilon g_{0}^{2}}+\frac{\tilde{\alpha}^{2} c_{0}}{\epsilon \theta_{1}^{2}}\right)\left|\frac{\rho_{1} k_{2}}{k_{1}}-\rho_{2}\right|\right\} .
$$

Therefore, from (3.82) and the definition of $E$, we obtain (3.64) for $\mathscr{L}_{2}$ instead of $\mathscr{L}_{1}$, and some $\tilde{c}_{8}>0$ (not depending on $\left.\int_{0}^{+\infty}|f(s)| d s\right)$ instead of $c_{8}$. Thus, according to (3.66) and under condition (3.8), where $\delta_{0}$ is defined in (3.67) with $\tilde{c}_{8}$ instead of $c_{8}$, we find, for some $\tilde{c}_{10}>0$,

$$
\mathscr{L}_{2}^{\prime}(t) \leq-\tilde{c}_{10} E(t)-\gamma(t) I_{7}(t) \leq-\tilde{c}_{10} E(t), \quad \forall t \in \mathbb{R}_{+} .
$$

Before concluding (3.11), we prove this last lemma.

Lemma 3.13. There exist two positive constants $a_{0}$ and $a_{1}$ such that, for any $\mathscr{U}_{0} \in \mathscr{D}\left(\mathscr{A}^{2}\right)$,

$$
\int_{S}^{T} E(t) d t \leq a_{1}\left(E(S)+E_{1}(S)\right), \quad \forall 0 \leq S \leq T
$$


and

$$
E^{\prime}(t) \leq a_{0} E(t), \quad \forall t \in \mathbb{R}_{+} .
$$

Proof. By integrating (3.86) over $[S, T]$, we get (note that $\mathscr{L}_{2} \geq 0$ in virtue of (3.84) and (3.85))

$$
\tilde{c}_{10} \int_{S}^{T} E(t) d t \leq \mathscr{L}_{2}(S)-\mathscr{L}_{2}(T) \leq \mathscr{L}_{2}(S), \quad \forall 0 \leq S \leq T .
$$

Moreover, (3.55) and (2.13) imply that

$$
\begin{aligned}
I_{7}(t) & \leq c_{0} \beta_{0}\left\|\eta_{s}(x, t, \cdot)\right\|_{L_{g}^{2}\left(\mathbb{R}_{+}, H_{0}^{1}(] 0, L[)\right)}^{2} \\
& \leq c_{0} \beta_{0}\left\|\psi_{t}(x, t)-\eta_{t}(x, t, \cdot)\right\|_{L_{g}^{2}\left(\mathbb{R}_{+}, H_{0}^{1}(j 0, L[))\right.}^{2} \\
& \leq 2 c_{0} \beta_{0}\left(\left\|\psi_{t}(x, t)\right\|_{L_{g}^{2}\left(\mathbb{R}_{+}, H_{0}^{1}(j 0, L \mathrm{D})\right.}^{2}+\left\|\eta_{t}(x, t, \cdot)\right\|_{L_{g}^{2}\left(\mathbb{R}_{+}, H_{0}^{1}(j 0, L[))\right.}^{2}\right) \\
& \leq 2 c_{0} \beta_{0}\left(g_{0} \int_{0}^{L} \psi_{x t}^{2}(x, t) d x+\int_{0}^{L} \int_{0}^{+\infty} g(s) \eta_{x t}^{2}(x, t, s) d s d x\right),
\end{aligned}
$$

and then, according to (3.71) for $k=1$,

$$
I_{7}(t) \leq 4 c_{0} \beta_{0} \max \left\{\frac{g_{0}}{k_{2}-g_{0}}, 1\right\} E_{1}(t) .
$$

Consequently, by substituting (3.90) and the right inequality of (3.84) in (3.89), we deduce (3.87) with

$$
a_{1}=\frac{1}{\tilde{c}_{10}} \max \left\{N+c_{7}+\tilde{c}_{7}, \frac{1}{\epsilon g_{0} \theta_{1}}\left|\frac{\rho_{1} k_{2}}{k_{1}}-\rho_{2}\right|+4 c_{0} \beta_{0} \max \left\{\frac{g_{0}}{k_{2}-g_{0}}, 1\right\}\right\} .
$$

On the other hand, taking (3.31) and (2.14) in consideration, integrating with respect to $s$ and using the fact that $\eta(x, t, 0)=\lim _{s \rightarrow+\infty} f(s) \eta(x, t, s)=0$ (thanks to (2.3), (2.9), (2.13), and (2.16) with $n$ $\geq 1$ ), we find

$$
\begin{aligned}
E^{\prime}(t) & \leq-\int_{0}^{L} \psi_{t}(x, t) \int_{0}^{+\infty} f(s) \psi_{t}(x, t-s) d s d x \\
& \leq-\int_{0}^{L} \psi_{t}(x, t) \int_{0}^{+\infty} f(s) \eta_{s}(x, t, s) d s d x \\
& \leq \int_{0}^{L} \psi_{t}(x, t) \int_{0}^{+\infty} f^{\prime}(s) \eta(x, t, s) d s d x,
\end{aligned}
$$

hence, applying Young's inequality and using (2.3) and (3.32) (for $v=|\eta|$ ) and (2.9),

$$
E^{\prime}(t) \leq \frac{1}{2} \int_{0}^{L} \psi_{t}^{2}(x, t) d x+\frac{\alpha^{2} g_{0} c_{0}}{2} \int_{0}^{L} \int_{0}^{+\infty} g(s) \eta_{x}^{2}(x, t, s) d s d x
$$

which gives (3.88) with $a_{0}=\max \left\{\frac{1}{\rho_{2}}, \alpha^{2} g_{0} c_{0}\right\}$.

Lemma 3.13 allows us to apply [Theorem 2.2 of Ref. 13: case $f \equiv 1, m=1, a_{2}=0$, and $n \geq 2$ ] and get (3.11) by continuity of $E$.

\section{Equal speed propagation and arbitrary decay of g: (1.2) and (3.3) hold, and (3.2) does not hold}

We prove here the decay estimate (3.12) under condition (3.3), which allows $g$ to have a general decay at infinity that can be arbitrary close to $\frac{1}{t}$.

In the cases of absence of delay and/or presence of frictional damping considered in the literature (like (1.3)), the proof of the known stability estimates when $g$ has an arbitrary decay is based on 
some differential inequalities on $g$ involving at least its first derivative in order to express

$$
\int_{0}^{L} \int_{0}^{+\infty} g(s) \eta_{x}^{2}(x, t, s) d s d x
$$

in term of

$$
\int_{0}^{L} \int_{0}^{+\infty} g^{\prime}(s) \eta_{x}^{2}(x, t, s) d s d x
$$

and then use the nonincreasingness of $E$. This strategy seems not applicable in our case, since $E$ is not necessarily nonincreasing due to the last term in (3.31) generated by the distributed time delay.

Our proof is based on different manipulations of the term (3.91), the integral inequality in (3.3) introduced and used in Refs. 36-39, and 40, which does not involve any derivative of $g$, and the use of a new functional $J_{6}$ (defined in (3.100) below) that is able to absorb some memory terms without passing by $E^{\prime}$.

First, following the idea in Refs. 36-39, and 40, we see that

$$
\begin{gathered}
2 \int_{0}^{L} \psi_{x}(x, t) \int_{0}^{+\infty} g(s) \psi_{x}(x, t-s) d s d x \\
=\int_{0}^{L} \int_{0}^{+\infty} g(s)\left(\psi_{x}^{2}(x, t-s)-\eta_{x}^{2}(x, t, s)\right) d s d x+g_{0} \int_{0}^{L} \psi_{x}^{2}(x, t) d x .
\end{gathered}
$$

Second, for $i \in \mathbb{N}^{*}$, we consider, as in Ref. 33, the set

$$
A_{i}=\left\{s \in \mathbb{R}_{+}, g(s) \leq-i g^{\prime}(s)\right\},
$$

and we put

$$
g_{i}=\int_{A_{i}^{c}} g(s) d s .
$$

Note that $g_{i}>0$, otherwise, $A_{i}^{c}=\emptyset$ and then (3.2) is satisfied for $\theta_{1}=\frac{1}{i}$, which is the case of exponential decay of $g$ treated previously. On the other hand, thanks to the second inequality in (3.3), we have $\lim _{i \rightarrow+\infty} A_{i}^{c}=\cap_{i \in \mathbb{N}^{*}} A_{i}^{c}=\emptyset$, and then

$$
\lim _{i \rightarrow+\infty} g_{i}=0
$$

Next, we go back to (3.35), (3.37), (3.42), and (3.51). Clearly, we have

$$
\begin{aligned}
\int_{0}^{L} \psi_{x}(x, t) \int_{0}^{+\infty} g(s) \eta_{x}(x, t, s) d s d x= & \int_{0}^{L} \psi_{x}(x, t) \int_{A_{i}} g(s) \eta_{x}(x, t, s) d s d x \\
& +\int_{0}^{L} \psi_{x}(x, t) \int_{A_{i}^{c}} g(s) \eta_{x}(x, t, s) d s d x .
\end{aligned}
$$

Then, using Cauchy-Schwarz and Young's inequalities for the two terms in the right hand side of the above equality and the definition (3.94) of $g_{i}$, we have, for any $\varepsilon_{2}>0$,

$$
\begin{aligned}
\int_{0}^{L} \psi_{x}(x, t) \int_{0}^{+\infty} g(s) \eta_{x}(x, t, s) d s d x \leq & \epsilon_{2} \int_{0}^{L} \psi_{x}^{2}(x, t) d x+\frac{g_{0}}{4 \epsilon_{2}} \int_{0}^{L} \int_{A_{i}} g(s) \eta_{x}^{2}(x, t, s) d s d x \\
& +\frac{\sqrt{g_{i}}}{2}\left(\int_{0}^{L} \psi_{x}^{2}(x, t) d x+\int_{0}^{L} \int_{A_{i}^{c}} g(s) \eta_{x}^{2}(x, t, s) d s d x\right) .
\end{aligned}
$$

Using the definition (3.93) of $A_{i}$, we obtain

$$
\int_{0}^{L} \psi_{x}(x, t) \int_{0}^{+\infty} g(s) \eta_{x}(x, t, s) d s d x
$$




$$
\begin{aligned}
\leq & \epsilon_{2} \int_{0}^{L} \psi_{x}^{2}(x, t) d x-\frac{i g_{0}}{4 \epsilon_{2}} \int_{0}^{L} \int_{0}^{+\infty} g^{\prime}(s) \eta_{x}^{2}(x, t, s) d s d x \\
& +\frac{\sqrt{g_{i}}}{2}\left(\int_{0}^{L} \psi_{x}^{2}(x, t) d x+\int_{0}^{L} \int_{0}^{+\infty} g(s) \eta_{x}^{2}(x, t, s) d s d x\right) .
\end{aligned}
$$

Similarly, we find

$$
\begin{gathered}
\int_{0}^{L}\left(\int_{0}^{+\infty} g(s) \eta_{x}(x, t, s) d s\right)^{2} d x \\
=\int_{0}^{L}\left(\int_{A_{i}} g(s) \eta_{x}(x, t, s) d s+\int_{A_{i}^{c}} g(s) \eta_{x}(x, t, s) d s\right)^{2} d x \\
\leq 2 \int_{0}^{L}\left(\int_{A_{i}} g(s) \eta_{x}(x, t, s) d s\right)^{2} d x+2 \int_{0}^{L}\left(\int_{A_{i}^{c}} g(s) \eta_{x}(x, t, s) d s\right)^{2} d x \\
\leq 2 g_{0} \int_{0}^{L} \int_{A_{i}} g(s) \eta_{x}^{2}(x, t, s) d s d x+2 g_{i} \int_{0}^{L} \int_{A_{i}^{c}} g(s) \eta_{x}^{2}(x, t, s) d s d x .
\end{gathered}
$$

Therefore, using again the definition (3.93) of $A_{i}$, we deduce that

$$
\begin{aligned}
\int_{0}^{L}\left(\int_{0}^{+\infty} g(s) \eta_{x}(x, t, s) d s\right)^{2} d x \leq & -2 i g_{0} \int_{0}^{L} \int_{0}^{+\infty} g^{\prime}(s) \eta_{x}^{2}(x, t, s) d s d x \\
& +2 g_{i} \int_{0}^{L} \int_{0}^{+\infty} g(s) \eta_{x}^{2}(x, t, s) d s d x
\end{aligned}
$$

The last term we discuss, using (3.92), is

$$
\begin{gathered}
-\int_{0}^{L} \psi_{x}(x, t) \int_{0}^{+\infty} g(s) \eta_{x}(x, t, s) d s d x \\
=-g_{0} \int_{0}^{L} \psi_{x}^{2}(x, t) d x+\int_{0}^{L} \psi_{x}(x, t) \int_{0}^{+\infty} g(s) \psi_{x}(x, t-s) d s d x \\
=-\frac{g_{0}}{2} \int_{0}^{L} \psi_{x}^{2}(x, t) d x+\frac{1}{2} \int_{0}^{L} \int_{0}^{+\infty} g(s)\left(\psi_{x}^{2}(x, t-s)-\eta_{x}^{2}(x, t, s)\right) d s d x .
\end{gathered}
$$

After, we insert (3.96), (3.97), and (3.98) into (3.35), (3.37), (3.42), and (3.51) considering the functional $\mathscr{L}_{1}$ defined in (3.57), using (3.31), (3.38), (3.41), and (3.54) taking (2.1) in consideration, choosing $\delta=\frac{k_{1}}{8 N_{1}}$ and

$$
0<\epsilon<\left\{\frac{k_{1}^{2}}{4 c_{3}}, \frac{k_{1} \rho_{1}}{8\left(2 k_{1}+c_{3}\right)}\right\}
$$

we obtain, for some $c_{11}, c_{12}>0$ (not depending on $N, N_{1}, N_{2}, i, \varepsilon_{1}, \varepsilon_{2}$ and $f$ ),

$$
\mathscr{L}_{1}^{\prime}(t)
$$




$$
\begin{aligned}
\leq & -\left(\frac{k_{2} N_{2}+g_{0}^{2} N_{1}}{2}-c_{11}-\frac{\varepsilon_{2}}{8}\right) \int_{0}^{L} \psi_{x}^{2}(x, t) d x-\frac{k_{1}}{8} \int_{0}^{L}\left(\varphi_{x}(x, t)+\psi(x, t)\right)^{2} d x \\
& -\left(\frac{\rho_{1}}{16}-\varepsilon_{1} N_{2}\right) \int_{0}^{L} \varphi_{t}^{2}(x, t) d x-\left(\rho_{2}\left(g_{0} N_{1}-\frac{k_{1}}{8}\right)-\frac{c_{6} N_{2}}{\varepsilon_{1}}-c_{11}\right) \int_{0}^{L} \psi_{t}^{2}(x, t) d x \\
& +\int_{0}^{L} \int_{0}^{+\infty}\left(-c_{N_{1}, N_{2}} g(s) \eta_{x}^{2}(x, t, s)+\left(\frac{N}{2}-c_{N_{1}, \varepsilon_{2}, i}\right) g^{\prime}(s) \eta_{x}^{2}(x, t, s)\right) d s d x \\
& -\int_{0}^{L} \int_{0}^{+\infty}|f(s)| \psi_{t}^{2}(x, t-s) d s d x+\frac{\sqrt{g_{i}}}{16} \int_{0}^{L} \psi_{x}^{2}(x, t) d x-\gamma(t) I_{7}(t) \\
& +c_{N_{1}}\left(g_{i}+\sqrt{g_{i}}\right) \int_{0}^{L} \int_{0}^{+\infty} g(s) \eta_{x}^{2}(x, t, s) d s d x \\
& +\left(\frac{g_{0} N_{1}+N_{2}}{2}+c_{12}\right) \int_{0}^{L} \int_{0}^{+\infty} g(s) \psi_{x}^{2}(x, t-s) d s d x \\
& +\int_{0}^{L} J_{5}(x, t) \int_{0}^{+\infty} f(s) \psi_{t}(x, t-s) d s d x+\left(\frac{\rho_{1} k_{2}}{k_{1}}-\rho_{2}\right) \int_{0}^{L} \varphi_{t}(x, t) \psi_{x t}(x, t) d x .
\end{aligned}
$$

Last, we introduce the functional $J_{6}$ and prove a crucial identity on its derivative.

Lemma 3.14. Let

$$
J_{6}(t)=\int_{0}^{L} \int_{0}^{t}\left(\int_{t}^{+\infty} g(\tau-s) d \tau\right) \psi_{x}^{2}(x, s) d s d x
$$

Then, for any $\lambda \in] 0,1[$,

$$
\begin{aligned}
J_{6}^{\prime}(t) \leq & -(1-\lambda) \xi(t) J_{6}(t)-\lambda \int_{0}^{L} \int_{0}^{+\infty} g(s) \psi_{x}^{2}(x, t-s) d s d x \\
& +g_{0} \int_{0}^{L} \psi_{x}^{2}(x, t) d x+\lambda \int_{0}^{L} \int_{t}^{+\infty} g(s) \psi_{0 x}^{2}(x, s-t) d s d x .
\end{aligned}
$$

Proof. First, thanks to the first inequality in (3.3) and the fact that $\eta \in L_{g}^{2}\left(\mathbb{R}_{+}, H_{0}^{1}(] 0, L[)\right)$ (due to (2.16) for $n=1$ ), we have

$$
\begin{aligned}
J_{6}(t) & \leq \frac{1}{\xi(t)} \int_{0}^{L} \int_{0}^{t} g(t-s) \psi_{x}^{2}(x, s) d s d x \\
& \leq \frac{1}{\xi(t)} \int_{0}^{L} \int_{0}^{t} g(s) \psi_{x}^{2}(x, t-s) d s d x \\
& \leq \frac{2}{\xi(t)}\left(g_{0} \int_{0}^{L} \psi_{x}^{2}(x, t) d x+\int_{0}^{L} \int_{0}^{+\infty} g(s) \eta_{x}^{2}(x, t, s) d s d x\right),
\end{aligned}
$$

hence, according to the definition of $E$,

$$
J_{6}(t) \leq \frac{4}{\xi(t)} \max \left\{\frac{g_{0}}{k_{2}-g_{0}}, 1\right\} E(t)
$$


Consequently, the functional $J_{6}$ is well-defined. Moreover, by the first inequality in (3.3), a simple and direct differentiation gives

$$
\begin{aligned}
J_{6}^{\prime}(t)= & \left(\int_{t}^{+\infty} g(\tau-t) d \tau\right) \int_{0}^{L} \psi_{x}^{2}(x, t) d x-\int_{0}^{L} \int_{0}^{t} g(t-s) \psi_{x}^{2}(x, s) d s d x \\
= & g_{0} \int_{0}^{L} \psi_{x}^{2}(x, t) d x-(1-\lambda) \int_{0}^{L} \int_{0}^{t} g(t-s) \psi_{x}^{2}(x, s) d s d x \\
& -\lambda \int_{0}^{L} \int_{-\infty}^{t} g(t-s) \psi_{x}^{2}(x, s) d s d x+\lambda \int_{0}^{L} \int_{-\infty}^{0} g(t-s) \psi_{x}^{2}(x, s) d s d x \\
\leq & g_{0} \int_{0}^{L} \psi_{x}^{2}(x, t) d x-(1-\lambda) \xi(t) J_{6}(t) \\
& -\lambda \int_{0}^{L} \int_{0}^{+\infty} g(s) \psi_{x}^{2}(x, t-s) d s d x+\lambda \int_{0}^{L} \int_{t}^{+\infty} g(s) \psi_{0 x}^{2}(x, s-t) d s d x,
\end{aligned}
$$

which is exactly (3.101).

Finally, let $N_{3}>0$ and

$$
\mathscr{F}_{1}(t):=\mathscr{L}_{1}(t)+N_{3} J_{6}(t) .
$$

Due to (1.2), the last term in (3.99) vanishes. Taking into account the relations (3.66), (3.99), and (3.101) we get

$$
\begin{gathered}
\mathscr{F}_{1}^{\prime}(t) \\
\leq-\left(\frac{k_{2} N_{2}+g_{0}^{2} N_{1}}{2}-c_{11}-g_{0} N_{3}-\frac{\varepsilon_{2}}{8}\right) \int_{0}^{L} \psi_{x}^{2}(x, t) d x-\left(\frac{\rho_{1}}{16}-\varepsilon_{1} N_{2}\right) \int_{0}^{L} \varphi_{t}^{2}(x, t) d x \\
-\frac{k_{1}}{8} \int_{0}^{L}\left(\varphi_{x}(x, t)+\psi(x, t)\right)^{2} d x-\left(\rho_{2}\left(g_{0} N_{1}-\frac{k_{1}}{8}\right)-\frac{c_{6} N_{2}}{\varepsilon_{1}}-c_{11}\right) \int_{0}^{L} \psi_{t}^{2}(x, t) d x \\
-c_{N_{1}, N_{2}} \int_{0}^{L} \int_{0}^{+\infty} g(s) \eta_{x}^{2}(x, t, s) d s d x+\left(\frac{N}{2}-c_{N_{1}, \varepsilon_{2}, i}\right) \int_{0}^{L} \int_{0}^{+\infty} g^{\prime}(s) \eta_{x}^{2}(x, t, s) d s d x \\
-\gamma(t) I_{7}(t)-(1-\lambda) N_{3} \xi(t) J_{6}(t)+\frac{\sqrt{g_{i}}}{16} \int_{0}^{L} \psi_{x}^{2}(x, t) d x \\
+c_{N_{1}}\left(g_{i}+\sqrt{g_{i}}\right) \int_{0}^{L} \int_{0}^{+\infty} g(s) \eta_{x}^{2}(x, t, s) d s d x+\lambda N_{3} \int_{0}^{L} \int_{t}^{+\infty} g(s) \psi_{0 x}^{2}(x, s-t) d s d x \\
+\frac{c_{9}}{4}\left(\int_{0}^{+\infty}|f(s)| d s\right) E(t)-\left(\lambda N_{3}-\frac{g_{0} N_{1}+N_{2}}{2}-c_{12}\right) \int_{0}^{L} \int_{0}^{+\infty} g(s) \psi_{x}^{2}(x, t-s) d s d x .
\end{gathered}
$$

Now, we choose the different constants carefully so as to obtain some desired signs of the coefficients. First, we select

$$
N_{2}>\frac{2}{k_{2}-g_{0}}\left(c_{11}+g_{0} c_{12}+1\right)
$$

(note that $N_{2}$ exists according to (2.1)). Next, we pick $\varepsilon_{1}$ such that

$$
0<\varepsilon_{1}<\min \left\{1, \frac{\rho_{1}}{16 N_{2}}\right\} \text {. }
$$

Then, we choose $N_{1}$ such that

$$
N_{1}>\frac{1}{\rho_{2} g_{0}}\left(c_{11}+\frac{c_{6} N_{2}}{\varepsilon_{1}}\right)+\frac{k_{1}}{8 g_{0}}
$$


$\left(N_{1}\right.$ exists due to (3.1)) and

$$
0<\varepsilon_{2}<8\left(\frac{\left(k_{2}-g_{0}\right) N_{2}}{2}-c_{11}-g_{0} c_{12}-1\right)
$$

(clearly $\varepsilon_{2}$ exists by our choice of $N_{2}$ ). Next, we select $N_{3}$ and $\lambda$ such that

$$
N_{3}=\frac{g_{0} N_{1}+N_{2}}{2}+c_{12}+\frac{1}{g_{0}} \text { and } \lambda=\frac{1}{N_{3}}\left(\frac{g_{0} N_{1}+N_{2}}{2}+c_{12}\right)
$$

(noting that $N_{3}$ exists thanks to (3.1), and $\left.\lambda \in\right] 0,1\left[\right.$ according to the choice of $N_{3}$ ). These choices imply that, for some $c_{13}, c_{14}>0$ (not depending on $N, i$ and $f$ ),

$$
\begin{aligned}
\mathscr{F}_{1}^{\prime}(t) \leq & -c_{13} E(t)-\gamma(t) I_{7}(t)-c_{13} \xi(t) J_{6}(t) \\
& +c_{14} \int_{0}^{L} \int_{t}^{+\infty} g(s) \psi_{0 x}^{2}(x, s-t) d s d x+\frac{c_{9}}{4}\left(\int_{0}^{+\infty}|f(s)| d s\right) E(t) \\
& +c_{14}\left(g_{i}+\sqrt{g_{i}}\right) E(t)+\left(\frac{N}{2}-\tilde{c}_{i}\right) \int_{0}^{L} \int_{0}^{+\infty} g^{\prime}(s) \eta_{x}^{2}(x, t, s) d s d x
\end{aligned}
$$

where $\tilde{c}_{i}$ is a positive constant depending on $i$. Then, under condition (3.8), where $\delta_{0}$ is defined in (3.67) with $c_{13}$ instead of $c_{8}$, and in virtue of (3.95), we can choose $i$ big enough so that

$$
g_{i}+\sqrt{g_{i}}<\frac{1}{c_{14}}\left(c_{13}-\frac{c_{9}}{4} \int_{0}^{+\infty}|f(s)| d s\right) .
$$

Last, we select $N$ big enough so that

$$
N>\max \left\{2 \tilde{c}_{i}, c_{7}\right\}
$$

where $c_{7}$ is defined in (3.61); so the last term in (3.105) is nonpositive and, using (3.62) and (3.103),

$$
\left(N-c_{7}\right) E(t) \leq \mathscr{F}_{1}(t)-I_{7}(t)-N_{3} J_{6}(t) \leq\left(N+c_{7}\right) E(t), \quad \forall t \in \mathbb{R}_{+} .
$$

According to our choices of $i$ and $N$, we deduce from (3.105) that, for some $c_{15}>0$,

$$
\mathscr{F}_{1}^{\prime}(t) \leq-c_{15} E(t)-\gamma(t) I_{7}(t)-c_{13} \xi(t) J_{6}(t)+c_{14} \int_{0}^{L} \int_{t}^{+\infty} g(s) \psi_{0 x}^{2}(x, s-t) d s d x .
$$

Therefore, using (3.106) and (3.107), we find, for

$$
\begin{gathered}
\delta_{1}=\min \left\{\frac{c_{15}}{N+c_{7}}, 1, \frac{c_{13}}{N_{3}}\right\}, \\
\mathscr{F}_{1}^{\prime}(t) \leq-\delta_{1} \min \{1, \gamma(t), \xi(t)\} \mathscr{F}_{1}(t)+c_{14} \int_{0}^{L} \int_{t}^{+\infty} g(s) \psi_{0 x}^{2}(x, s-t) d s d x .
\end{gathered}
$$

By integrating the differential inequality (3.108) over $[0, t]$, we get

$$
\mathscr{F}_{1}(t) \leq e^{-\delta_{1} \phi(t)}\left(\mathscr{F}_{1}(0)+c_{14} \int_{0}^{L} \int_{0}^{t} e^{\delta_{1} \phi(s)} \int_{s}^{+\infty} g(\tau) \psi_{0 x}^{2}(x, \tau-s) d \tau d s d x\right),
$$

where $\phi$ is defined in (3.13). Then, from (3.30), (3.106), and (3.109) we find

$$
\begin{aligned}
\|\mathscr{U}(t)\|_{\mathscr{H}}^{2} & =2 E(t) \\
& \leq \frac{2}{N-c_{7}} \mathscr{F}_{1}(t) \\
& \leq \delta_{2} e^{-\delta_{1} \phi(t)}\left(1+\int_{0}^{L} \int_{0}^{t} e^{\delta_{1} \phi(s)} \int_{s}^{+\infty} g(\tau) \psi_{0 x}^{2}(x, \tau-s) d \tau d s d x\right),
\end{aligned}
$$

which gives (3.12) with $\delta_{2}=\frac{2}{N-c_{7}} \max \left\{c_{14}, \mathscr{F}_{1}(0)\right\}$. 


\section{APPLICATIONS}

Our well-posedness and asymptotic stability results for (1.1) hold for various Timoshenko-type systems. We present here some examples.

\section{A. Timoshenko-heat}

Let us consider coupled Timoshenko-heat systems under Fourier's law of heat conduction

$$
\left\{\begin{array}{l}
\rho_{1} \varphi_{t t}(x, t)-k_{1}\left(\varphi_{x}(x, t)+\psi(x, t)\right)_{x}=0, \\
\rho_{2} \psi_{t t}(x, t)-k_{2} \psi_{x x}(x, t)+k_{1}\left(\varphi_{x}(x, t)+\psi(x, t)\right)+k_{4} \theta_{x}(x, t) \\
\quad \quad \quad \int_{0}^{+\infty} g(s) \psi_{x x}(x, t-s) d s+\int_{0}^{+\infty} f(s) \psi_{t}(x, t-s) d s=0, \\
\rho_{3} \theta_{t}(x, t)-k_{3} \theta_{x x}(x, t)+k_{4} \psi_{x t}(x, t)=0, \\
\varphi(0, t)=\psi(0, t)=\theta(0, t)=\varphi(L, t)=\psi(L, t)=\theta(L, t)=0, \\
\varphi(x, 0)=\varphi_{0}(x), \quad \varphi_{t}(x, 0)=\varphi_{1}(x), \\
\psi(x,-t)=\psi_{0}(x, t), \quad \psi_{t}(x,-t)=\psi_{1}(x, t), \quad \theta(x, 0)=\theta_{0}(x),
\end{array}\right.
$$

where $\theta$ denotes the temperature difference (see Ref. 9 for more details).

Under (A1) and (A2), system (4.1) can be formulated in the abstract form (2.4), where $\mathscr{U}=$ $\left(\varphi, \psi, \varphi_{t}, \psi_{t}, \theta, \eta\right)^{T}, \mathscr{U}_{0}=\left(\varphi_{0}, \psi_{0}(\cdot, 0), \varphi_{1}, \psi_{1}(\cdot, 0), \theta_{0}, \eta_{0}\right)^{T} \in \mathscr{H}$,

$$
\mathscr{H}=\left(H_{0}^{1}(] 0, L[)\right)^{2} \times\left(L^{2}(] 0, L[)\right)^{3} \times L_{g}^{2}\left(\mathbb{R}_{+}, H_{0}^{1}(] 0, L[)\right),
$$

and the operators $\mathscr{A}$ and $\mathscr{B}$ are given by ( $\varepsilon_{0}$ and $c_{0}$ are defined, respectively, in (2.8) and (2.9)),

$$
\mathscr{B}\left(w_{1}, w_{2}, w_{3}, w_{4}, w_{5}, w_{6}\right)^{T}=\left(0,0,0, \frac{\|f\|_{\infty}}{\rho_{2}} w_{4}, 0, \epsilon_{0} w_{6}\right)^{T}
$$

and

$$
\mathscr{A}\left(\begin{array}{l}
w_{1} \\
w_{2} \\
w_{3} \\
w_{4} \\
w_{5} \\
w_{6}
\end{array}\right)=\left(\begin{array}{c}
w_{3} \\
w_{4} \\
\frac{k_{1}}{\rho_{1}}\left(w_{1 x}+w_{2}\right)_{x} \\
\tilde{w}_{4} \\
\frac{1}{\rho_{3}}\left(k_{3} w_{5 x}-k_{4} w_{4}\right)_{x} \\
-w_{6 s}-\epsilon_{0} w_{6}+w_{4}
\end{array}\right),
$$

where

$$
\begin{aligned}
\tilde{w}_{4}= & \frac{1}{\rho_{2}}\left(k_{2}-g_{0}\right) w_{2 x x}-\frac{k_{1}}{\rho_{2}}\left(w_{1 x}+w_{2}\right)-\frac{k_{4}}{\rho_{2}} w_{5 x}-\frac{\|f\|_{\infty}}{\rho_{2}} w_{4} \\
& +\frac{1}{\rho_{2}} \int_{0}^{+\infty} g(s) w_{6 x x}(s) d s-\frac{1}{\rho_{2}} \int_{0}^{+\infty} f(s) w_{6 s}(s) d s .
\end{aligned}
$$

From (2.1), $\mathscr{H}$ endowed with the inner product, for $W=\left(w_{1}, w_{2}, w_{3}, w_{4}, w_{5}, w_{6}\right)^{T}$ and $\tilde{W}^{T}=$ $\left(\tilde{w}_{1}, \tilde{w}_{2}, \tilde{w}_{3}, \tilde{w}_{4}, \tilde{w}_{5}, \tilde{w}_{6}\right)^{T}$,

$$
\begin{aligned}
\langle W, \tilde{W}\rangle_{\mathscr{H}}= & \int_{0}^{L}\left(\left(k_{2}-g_{0}\right) w_{2 x}(x) \tilde{w}_{2 x}(x)+k_{1}\left(w_{1 x}(x)+w_{2}(x)\right)\left(\tilde{w}_{1 x}(x)+\tilde{w}_{2}(x)\right)\right) d x \\
& +\int_{0}^{L}\left(\rho_{1} w_{3}(x) \tilde{w}_{3}(x)+\rho_{2} w_{4}(x) \tilde{w}_{4}(x)+\rho_{3} w_{5}(x) \tilde{w}_{5}(x)\right) d x \\
& +\left\langle w_{6}, \tilde{w}_{6}\right\rangle_{L_{g}^{2}\left(\mathbb{R}_{+}, H_{0}^{1}(j 0, L \mathrm{D})\right)}
\end{aligned}
$$

is a Hilbert space. Similar to the proof of Theorem 2.1 for (1.1), we can prove that the linear operator $-\mathscr{A}$ is a maximal monotone operator, and $\mathscr{B}$ is Lipschitz continuous. Then $\mathscr{A}+\mathscr{B}$ is an 
infinitesimal generator of a linear $C_{0}$-semigroup on $\mathscr{H}$. Consequently, (2.4) associated with (4.1) is well-posed in the sense of Theorem 2.1.

On the other hand, under (A1)-(A4), Theorem 3.2 holds for (4.1). We use the same functionals and arguments given in Sec. III (see also Ref. 19 in case $f \equiv 0$ ).

\section{B. Timoshenko-thermoelasticity}

Our approach can be applied to the following Timoshenko-thermoelasticity system of type III,

$$
\left\{\begin{array}{l}
\rho_{1} \varphi_{t t}(x, t)-k_{1}\left(\varphi_{x}(x, t)+\psi(x, t)\right)_{x}=0, \\
\rho_{2} \psi_{t t}(x, t)-k_{2} \psi_{x x}(x, t)+k_{1}\left(\varphi_{x}(x, t)+\psi(x, t)\right)+k_{4} \theta_{x t}(x, t) \\
\quad \quad+\int_{0}^{+\infty} g(s) \psi_{x x}(x, t-s) d s+\int_{0}^{+\infty} f(s) \psi_{t}(x, t-s) d s=0, \\
\rho_{3} \theta_{t t}(x, t)-k_{3} \theta_{x x}(x, t)+k_{4} \psi_{x t}(x, t)-k_{5} \theta_{x x t}(x, t)=0, \\
\varphi(0, t)=\psi(0, t)=\theta_{x}(0, t)=\varphi(L, t)=\psi(L, t)=\theta_{x}(L, t)=0, \\
\varphi(x, 0)=\varphi_{0}(x), \quad \varphi_{t}(x, 0)=\varphi_{1}(x), \\
\psi(x,-t)=\psi_{0}(x, t), \quad \psi_{t}(x,-t)=\psi_{1}(x, t), \\
\theta(x, 0)=\theta_{0}(x), \quad \theta_{t}(x, 0)=\theta_{1}(x),
\end{array}\right.
$$

which models the transverse vibrations of a thick beam, taking into account the heat conduction introduced in Refs. 10-12.

Under (A1) and (A2), system (4.2) also can be formulated in the abstract form (2.4), where $\mathscr{U}=\left(\varphi, \psi, \theta, \varphi_{t}, \psi_{t}, \theta_{t}, \eta\right)^{T}, \mathscr{U}_{0}=\left(\varphi_{0}, \psi_{0}(\cdot, 0), \theta_{0}, \varphi_{1}, \psi_{1}(\cdot, 0), \theta_{1}, \eta_{0}\right)^{T} \in \mathscr{H}$,

$$
\begin{gathered}
\mathscr{H}=\left(H_{0}^{1}(] 0, L[)\right)^{2} \times H_{*}^{1}(] 0, L[) \times\left(L^{2}(] 0, L[)\right)^{2} \times L_{*}^{2}(] 0, L[) \times L_{g}^{2}\left(\mathbb{R}_{+}, H_{0}^{1}(] 0, L[)\right), \\
H_{*}^{1}(] 0, L[)=\left\{v \in H^{1}(] 0, L[): \int_{0}^{L} v(x) d x=0\right\}, \quad L_{*}^{2}(] 0, L[)=\left\{v \in L^{2}(] 0, L[): \int_{0}^{L} v(x) d x=0\right\}
\end{gathered}
$$

and the operators $\mathscr{A}$ and $\mathscr{B}$ are given by

$$
\mathscr{B}\left(w_{1}, w_{2}, w_{3}, w_{4}, w_{5}, w_{6}, w_{7}\right)^{T}=\left(0,0,0,0, \frac{\|f\|_{\infty}}{\rho_{2}} w_{5}, 0, \epsilon_{0} w_{7}\right)^{T}
$$

and

$$
\mathscr{A}\left(\begin{array}{l}
w_{1} \\
w_{2} \\
w_{3} \\
w_{4} \\
w_{5} \\
w_{6} \\
w_{7}
\end{array}\right)=\left(\begin{array}{c}
w_{4} \\
w_{5} \\
w_{6} \\
\frac{k_{1}}{\rho_{1}}\left(w_{1 x}+w_{2}\right)_{x} \\
\tilde{w}_{5} \\
\frac{1}{\rho_{3}}\left(k_{3} w_{3 x}-k_{4} w_{5}+k_{5} w_{6 x}\right)_{x} \\
-w_{7 s}-\epsilon_{0} w_{7}+w_{5}
\end{array}\right),
$$

where

$$
\begin{aligned}
\tilde{w}_{5}= & \frac{1}{\rho_{2}}\left(k_{2}-g_{0}\right) w_{2 x x}-\frac{k_{1}}{\rho_{2}}\left(w_{1 x}+w_{2}\right)-\frac{k_{4}}{\rho_{2}} w_{6 x}-\frac{\|f\|_{\infty}}{\rho_{2}} w_{5} \\
& +\frac{1}{\rho_{2}} \int_{0}^{+\infty} g(s) w_{7 x x}(s) d s-\frac{1}{\rho_{2}} \int_{0}^{+\infty} f(s) w_{7 s}(s) d s .
\end{aligned}
$$


From (2.1), $\mathscr{H}$ endowed with the inner product, for $W=\left(w_{1}, w_{2}, w_{3}, w_{4}, w_{5}, w_{6}, w_{7}\right)^{T}$ and $\tilde{W}=$ $\left(\tilde{w}_{1}, \tilde{w}_{2}, \tilde{w}_{3}, \tilde{w}_{4}, \tilde{w}_{5}, \tilde{w}_{6}, \tilde{w}_{7}\right)^{T}$,

$$
\begin{aligned}
\langle W, \tilde{W}\rangle_{\mathscr{H}}= & \int_{0}^{L}\left(\left(k_{2}-g_{0}\right) w_{2 x}(x) \tilde{w}_{2 x}(x)+k_{1}\left(w_{1 x}(x)+w_{2}(x)\right)\left(\tilde{w}_{1 x}(x)+\tilde{w}_{2}(x)\right)\right) d x \\
& +\int_{0}^{L}\left(k_{3} w_{3 x}(x) \tilde{w}_{3 x}(x)+\rho_{1} w_{4}(x) \tilde{w}_{4}(x)+\rho_{2} w_{5}(x) \tilde{w}_{5}(x)+\rho_{3} w_{6}(x) \tilde{w}_{6}(x)\right) d x \\
& +\left\langle w_{7}, \tilde{w}_{7}\right\rangle_{L_{g}^{2}\left(\mathbb{R}_{+}, H_{0}^{1}(] 0, L[)\right)}
\end{aligned}
$$

is a Hilbert space. Similar to the proof of Theorem 2.1 for $(1.1)$, we can prove that $\mathscr{A}+\mathscr{B}$ is an infinitesimal generator of a linear $C_{0}$-semigroup on $\mathscr{H}$ by proving that $-\mathscr{A}$ is maximal monotone and $\mathscr{B}$ is Lipschitz continuous, and then we deduce that (2.4) associated with (4.2) is well-posed in the sense of Theorem 2.1 .

On the other hand, under (A1)-(A4), Theorem 3.2 holds for (4.2), where, here, $E(t)=$ $\frac{1}{2}\left\|\left(\varphi, \psi, \tilde{\theta}, \varphi_{t}, \psi_{t}, \tilde{\theta}_{t}, \eta\right)\right\|_{\mathscr{H}}^{2}$ and

$$
\tilde{\theta}(x, t)=\theta(x, t)-\frac{t}{L} \int_{0}^{L} \theta_{1}(y) d y-\frac{1}{L} \int_{0}^{L} \theta_{0}(y) d y
$$

so

$$
\int_{0}^{L} \tilde{\theta}(x, t) d x=0,
$$

and then (2.9) is applicable for $\tilde{\theta}$. For the proof, we use the same functionals as in Sec. III and some arguments in Ref. 19 considered in case $f \equiv 0$.

\section{Porous thermoelastic}

Our approach can also be applied to the following porous thermoelastic system:

$$
\left\{\begin{array}{l}
\rho_{1} \varphi_{t t}(x, t)-k_{1}\left(\varphi_{x}(x, t)+\psi(x, t)\right)_{x}+k_{4} \theta_{x}(x, t)=0, \\
\rho_{2} \psi_{t t}(x, t)-k_{2} \psi_{x x}(x, t)+k_{1}\left(\varphi_{x}(x, t)+\psi(x, t)\right)-k_{5} \theta(x, t) \\
\quad+\int_{0}^{+\infty} g(s) \psi_{x x}(x, t-s) d s+\int_{0}^{+\infty} f(s) \psi_{t}(x, t-s) d s=0, \\
\rho_{3} \theta_{t}(x, t)-k_{3} \theta_{x x}(x, t)+k_{4} \varphi_{x t}(x, t)+k_{5} \psi_{t}(x, t)=0, \\
\varphi(0, t)=\psi(0, t)=\theta(0, t)=\varphi(L, t)=\psi(L, t)=\theta(L, t)=0, \\
\varphi(x, 0)=\varphi_{0}(x), \quad \varphi_{t}(x, 0)=\varphi_{1}(x), \\
\psi(x,-t)=\psi_{0}(x, t), \quad \psi_{t}(x,-t)=\psi_{1}(x, t), \quad \theta(x, 0)=\theta_{0}(x) .
\end{array}\right.
$$

Under (A1) and (A2), system (4.3) can be formulated in the abstract form (2.4), where $\mathscr{U}, \mathscr{U}_{0}, \mathscr{B}$, and $\langle\cdot, \cdot\rangle_{\mathscr{H}}$ are defined as in case (4.1), and the operator $\mathscr{A}$ is given by

$$
\mathscr{A}\left(\begin{array}{c}
w_{1} \\
w_{2} \\
w_{3} \\
w_{4} \\
w_{5} \\
w_{6}
\end{array}\right)=\left(\begin{array}{c}
w_{3} \\
w_{4} \\
\frac{k_{1}}{\rho_{1}}\left(w_{1 x}+w_{2}\right)_{x}-\frac{k_{4}}{\rho_{1}} w_{5 x} \\
\tilde{w}_{4} \\
\frac{1}{\rho_{3}}\left(k_{3} w_{5 x}-k_{4} w_{3}\right)_{x}-\frac{k_{5}}{\rho_{3}} w_{4} \\
-w_{6 s}-\epsilon_{0} w_{6}+w_{4}
\end{array}\right),
$$

where

$$
\begin{aligned}
\tilde{w}_{4}= & \frac{1}{\rho_{2}}\left(k_{2}-g_{0}\right) w_{2 x x}-\frac{k_{1}}{\rho_{2}}\left(w_{1 x}+w_{2}\right)+\frac{k_{5}}{\rho_{2}} w_{5}-\frac{\|f\|_{\infty}}{\rho_{2}} w_{4} \\
& +\frac{1}{\rho_{2}} \int_{0}^{+\infty} g(s) w_{6 x x}(s) d s-\frac{1}{\rho_{2}} \int_{0}^{+\infty} f(s) w_{6 s}(s) d s .
\end{aligned}
$$


As in the previous applications, the proof of Theorem 2.1 for (4.3) is similar to the one given in Sec. II for (1.1).

Under (A1)-(A4), Theorem 3.2 holds also for (4.3) with the same proof as in Sec. III.

\section{Discrete time delay}

Similar well-posedness results to the ones in Theorem 2.1 and, under (1.2), the stability estimates (3.9) and (3.12) hold in the case of discrete time delay for (1.1) as well as for (4.1)-(4.3). Let us discuss the case of (1.1) with discrete time delay (the cases of (4.1)-(4.3) with discrete time delay can be treated similarly)

$$
\left\{\begin{array}{l}
\rho_{1} \varphi_{t t}(x, t)-k_{1}\left(\varphi_{x}(x, t)+\psi(x, t)\right)_{x}=0, \\
\rho_{2} \psi_{t t}(x, t)-k_{2} \psi_{x x}(x, t)+k_{1}\left(\varphi_{x}(x, t)+\psi(x, t)\right) \\
\quad \quad \quad \int_{0}^{+\infty} g(s) \psi_{x x}(x, t-s) d s+\mu \psi_{t}(x, t-\tau)=0, \\
\quad \varphi(0, t)=\psi(0, t)=\varphi(L, t)=\psi(L, t)=0, \\
\varphi(x, 0)=\varphi_{0}(x), \quad \varphi_{t}(x, 0)=\varphi_{1}(x), \\
\psi(x,-t)=\psi_{0}(x, t), \quad \psi_{t}(x, 0)=\psi_{1}(x), \quad \psi_{t}(x, t-\tau)=f_{0}(x, t-\tau)(t \in] 0, \tau[),
\end{array}\right.
$$

where $\tau \in] 0,+\infty\left[, \mu \in \mathbb{R}^{*}\right.$ and $\left(\varphi_{0}, \psi_{0}, \varphi_{1}, \psi_{1}, f_{0}\right)$ are given initial data.

We prove briefly that (4.4) is well posed under the assumption (A1), and it is stable provided that (1.2), (A1), and (A3) hold and $|\mu|$ is small enough, and we establish decay estimates similar to (3.9) and (3.12).

\section{Well-posedness}

Following the idea in Ref. 28 (see also Refs. 29-31) to deal with the delay term by considering a new auxiliary variable $z$, we can formulate the system (4.4) in the abstract form (2.4), where $\mathscr{U}=\left(\varphi, \psi, \varphi_{t}, \psi_{t}, \eta, z\right)^{T}, \mathscr{U}_{0}=\left(\varphi_{0}, \psi_{0}(\cdot, 0), \varphi_{1}, \psi_{1}, \eta_{0}, z_{0}\right)^{T} \in \mathscr{H}$,

$$
\begin{aligned}
& \mathscr{H}=\left(H_{0}^{1}(] 0, L[)\right)^{2} \times\left(L^{2}(] 0, L[)\right)^{2} \times L_{g}^{2}\left(\mathbb{R}_{+}, H_{0}^{1}(] 0, L[)\right) \times L^{2}(] 0,1\left[, L^{2}(] 0, L[)\right), \\
& L^{2}(] 0,1\left[, L^{2}(] 0, L[)\right)=\{w:] 0,1\left[\rightarrow L^{2}(] 0, L[), \quad \int_{0}^{L} \int_{0}^{1} w^{2}(x, p) d p d x<+\infty\right\}
\end{aligned}
$$

endowed with the inner product

$$
\left\langle w_{1}, w_{2}\right\rangle_{L^{2}\left(\left[0,1\left[, L^{2}(00, L[))\right.\right.\right.}=\int_{0}^{L} \int_{0}^{1} w_{1}(x, p) w_{2}(x, p) d p d x,
$$

and

$$
\left\{\begin{array}{l}
z(x, t, p)=\psi_{t}(x, t-\tau p), \\
z_{0}(x, p)=z(x, 0, p)=f_{0}(x,-\tau p) .
\end{array}\right.
$$

The operators $\mathscr{A}$ and $\mathscr{B}$ are linear and given by

$$
\mathscr{B}\left(w_{1}, w_{2}, w_{3}, w_{4}, w_{5}, w_{6}\right)^{T}=\frac{|\mu|}{\rho_{2}}\left(0,0,0, w_{4}, 0,0\right)^{T}
$$

and

$$
\mathscr{A}\left(\begin{array}{l}
w_{1} \\
w_{2} \\
w_{3} \\
w_{4} \\
w_{5} \\
w_{6}
\end{array}\right)=\left(\begin{array}{c}
w_{3} \\
w_{4} \\
\frac{k_{1}}{\rho_{1}}\left(w_{1 x}+w_{2}\right)_{x} \\
\tilde{w}_{4} \\
-w_{5 s}+w_{4} \\
-\frac{1}{\tau} w_{6 p}
\end{array}\right),
$$


where

$$
\begin{aligned}
\tilde{w}_{4}= & \frac{1}{\rho_{2}}\left(k_{2}-g_{0}\right) w_{2 x x}-\frac{k_{1}}{\rho_{2}}\left(w_{1 x}+w_{2}\right)-\frac{|\mu|}{\rho_{2}} w_{4} \\
& -\frac{\mu}{\rho_{2}} w_{6}(1)+\frac{1}{\rho_{2}} \int_{0}^{+\infty} g(s) w_{5 x x}(s) d s .
\end{aligned}
$$

The domains $\mathscr{D}(\mathscr{A})$ and $\mathscr{D}(\mathscr{B})$ of $\mathscr{A}$ and $\mathscr{B}$, respectively, are given by

$$
\mathscr{D}(\mathscr{A})=\left\{\begin{array}{c}
\left(w_{1}, w_{2}, w_{3}, w_{4}, w_{5}, w_{6}\right)^{T} \in \mathscr{H}, w_{5}(0)=0, w_{6}(0)=w_{4} \\
w_{6 p} \in L^{2}(] 0,1\left[, L^{2}(] 0, L[)\right), w_{5 s} \in L_{g}^{2}\left(\mathbb{R}_{+}, H_{0}^{1}(] 0, L[)\right), w_{3}, w_{4} \in H_{0}^{1}(] 0, L[) \\
w_{1} \in H^{2}(] 0, L[),\left(k_{2}-g_{0}\right) w_{2 x x}+\int_{0}^{+\infty} g(s) w_{5 x x}(s) d s \in L^{2}(] 0, L[)
\end{array}\right\}
$$

and $\mathscr{D}(\mathscr{B})=\mathscr{H}$. Keeping in mind the definition (4.5) of $z$, we have

$$
\left\{\begin{array}{l}
\tau z_{t}(x, t, p)+z_{p}(x, t, p)=0, \\
z(x, t, 0)=\psi_{t}(x, t) .
\end{array}\right.
$$

Therefore, we conclude from (2.13) and (4.10) that the systems (4.4) and (2.4) are equivalent.

Clearly, thanks to (2.1), $\mathscr{H}$ endowed with the inner product, for $W=\left(w_{1}, w_{2}, w_{3}, w_{4}, w_{5}, w_{6}\right)^{T}$ and $\tilde{W}=\left(\tilde{w}_{1}, \tilde{w}_{2}, \tilde{w}_{3}, \tilde{w}_{4}, \tilde{w}_{5}, \tilde{w}_{6}\right)^{T}$,

$$
\begin{aligned}
\langle W, \tilde{W}\rangle_{\mathscr{H}}= & \int_{0}^{L}\left(\left(k_{2}-g_{0}\right) w_{2 x}(x) \tilde{w}_{2 x}(x)+k_{1}\left(w_{1 x}(x)+w_{2}(x)\right)\left(\tilde{w}_{1 x}(x)+\tilde{w}_{2}(x)\right)\right) d x \\
& +\int_{0}^{L}\left(\rho_{1} w_{3}(x) \tilde{w}_{3}(x)+\rho_{2} w_{4}(x) \tilde{w}_{4}(x)\right) d x \\
& +\left\langle w_{5}, \tilde{w}_{5}\right\rangle_{\left.L_{g}^{2}\left(\mathbb{R}_{+}, H_{0}^{1}(] 0, L D\right)\right)}+\tau|\mu|\left\langle w_{6}, \tilde{w}_{6}\right\rangle_{L^{2}\left(00,1\left[L^{2}(] 0, L D\right)\right)}
\end{aligned}
$$

is a Hilbert space and $\mathscr{D}(\mathscr{A}) \subset \mathscr{H}$ with dense embedding. Similar to the proof of Theorem 2.1 for (1.1), we can prove that the linear operator $-\mathscr{A}$ is a maximal monotone operator, and $\mathscr{B}$ is Lipschitz continuous; the proof is similar to the one given in Ref. 15 for an abstract evolution equation with infinite memory and discrete time delay. Then $\mathscr{A}+\mathscr{B}$ is an infinitesimal generator of a linear $C_{0^{-}}$ semigroup on $\mathscr{H}$ (see Ref. 34: Chap. 3 - Theorem 1.1). Consequently, (2.4) associated with (4.4) is well-posed in the sense of Theorem 2.1 (see Refs. 22 and 34).

\section{Stability}

We prove here that the system (2.4) associated with (4.4) is stable under (A1), (A3), and (1.2), and provided that $|\mu|$ is small enough. We provide two decay estimates depending on $\xi$.

Theorem 4.1. Assume (A1), (A3), and (1.2) hold. Then there exists a positive constant $\delta_{0}$ independent of $\mu$ such that, if

$$
|\mu|<\delta_{0},
$$

then, for any $\mathscr{U}_{0} \in \mathscr{H}$, there exist positive constants $\delta_{1}$ and $\delta_{2}$ such that the weak solution of (2.4) associated with (4.4) satisfies (3.9) with $\phi(t)=t$ if (3.2) holds, and it satisfies (3.12) with $\phi(t)=\int_{0}^{t} \min \{1, \xi(s)\} d s$ if (3.2) does not hold and (3.3) holds.

Proof. Let $\mathscr{U}_{0} \in \mathscr{D}(\mathscr{A})$, so that all the calculations below are justified. By a simple density argument, the decay estimates in Theorem 4.1 remain valid for any weak solution $\left(\mathscr{U}_{0} \in \mathscr{H}\right)$. First, as in Ref. 15, we provide a bound on the derivative of the energy functional $E$ (defined in (3.30)) 
associated with the solution of (2.4). We find

$$
E^{\prime}(t) \leq \frac{1}{2} \int_{0}^{L} \int_{0}^{+\infty} g^{\prime}(s) \eta_{x}^{2}(x, t, s) d s d x+|\mu| \int_{0}^{L} \psi_{t}^{2}(x, t) d x .
$$

Note that, as in the distributed delay case, the sign of $E^{\prime}$ cannot be determined directly from (4.12).

The proof of Theorem 4.1 is identical to the one given for system (1.1). The only modification of the proof given in Sec. III for (3.9) and (3.12) is the use of the following functional $J_{7}$, introduced in Ref. 28, instead of $I_{7}$ (defined in (3.52)) to get a crucial estimate on the discrete delay term.

Lemma 4.2. The functional

$$
J_{7}(t)=\tau e^{2 \tau} \int_{0}^{L} \int_{0}^{1} e^{-2 \tau p} z^{2}(x, t, p) d p d x
$$

satisfies

$$
J_{7}^{\prime}(t)=-2 J_{7}(t)+e^{2 \tau} \int_{0}^{L} \psi_{t}^{2}(x, t) d x-\int_{0}^{L} z^{2}(x, t, 1) d x
$$

Proof. See, for example, Ref. 20.

Now, defining $\mathscr{L}_{1}$ and $\mathscr{F}_{1}$, respectively, by (3.57) and (3.103) with $J_{7}$ instead of $I_{7}$, we get, for some positive constants $\delta_{1}, c_{16}>0$ (as for (3.69) and (3.108)),

$$
\mathscr{L}_{1}^{\prime}(t) \leq-\delta_{1} \mathscr{L}_{1}(t), \quad \forall t \in \mathbb{R}_{+}
$$

when (3.2) holds, and

$$
\mathscr{F}_{1}^{\prime}(t) \leq-\delta_{1} \min \{1, \xi(t)\} \mathscr{F}_{1}(t)+c_{16} \int_{0}^{L} \int_{t}^{+\infty} g(s) \psi_{0 x}^{2}(x, t-s) d s d x, \quad \forall t \in \mathbb{R}_{+}
$$

when (3.2) does not hold and (3.3) holds. The rest of the proof carries out as in the case of distributed delay.

\section{GENERAL COMMENTS AND ISSUES}

1. In the case of distributed time delay (1.1) and (4.1)-(4.3), the decay estimates (3.9) and (3.12) are obtained only for classical solutions (that is, for $\mathscr{U}_{0} \in \mathscr{D}(\mathscr{A})$ ), since the functional $I_{7}$ defined in (3.52) is not well-defined for weak solutions; that is, when $\mathscr{U}_{0} \in \mathscr{H}$ (see (3.55)).

2. When (1.2) does not hold (which is more interesting from the physical point of view), proving the stability of (4.1)-(4.3) with discrete delay instead of the distributed one (that is, the infinite integral depending on $f$ is replaced by $\left.\mu \psi_{t}(x, t-\tau)\right)$ and (4.4), seems a delicate question, since the second energy $E_{1}$ (defined in (3.71) with $k=1$ ) satisfies

$$
E_{1}^{\prime}(t) \leq \frac{1}{2} \int_{0}^{L} \int_{0}^{+\infty} g^{\prime}(s) \eta_{x t}^{2}(x, t, s) d s d x+|\mu| \int_{0}^{L} \psi_{t t}^{2}(x, t) d x
$$

so $E_{1}$ is not necessarily nonincreasing due to the term depending on $\mu$, this term cannot be absorbed by $E$ itself even if $|\mu|$ is supposed small enough. In the case of distributed delay, the key of solution was the introduction of the functional $I_{8}$ defined in (3.78).

3. In the case of absence of delay (i.e., $f \equiv 0$ and $\mu=0$ ), it is well-known that (see Refs. 8,9, and 19), the systems (1.1), (4.1), (4.2), and (4.3) are not exponentially stable when (1.2) does not hold, but they are dissipative (with respect to $E$ ) and the energy satisfies some weaker decay estimates depending on the (exponential or arbitrary) decay of $g$ at infinity and the regularity of the initial data $\mathscr{U}_{0}$. In the particular case of exponential decay (3.2), the decay rate obtained in Refs. 8,9, and 19 is of type $\frac{1}{t^{n}}$, which is stronger than the one given in (3.11). This fact is caused by the nondissipativeness character of (1.1), (4.1), (4.2), and (4.3) generated by the presence of delay. 
4. In both distributed and discrete delay cases, the arguments presented in this paper can be adapted to the case where the delay is considered in the first equation; so similar well-posedness and stability results can be obtained. However, proving the stability of (1.1) in the case where the memory is considered in the first equation seems a delicate question.

5. The estimate (3.12) does not imply (3.29); that limit depends on the connection between the growths at infinity of $g, f$, and $\int_{0}^{L} \psi_{0 x}^{2}(x, \cdot) d x$.

If $\psi_{0 x}$ satisfies

$$
\int_{0}^{L} \int_{0}^{+\infty} \psi_{0 x}^{2}(x, s) d s d x<+\infty
$$

then (3.9) holds, where $\phi$ is defined in (3.13). The idea of proof relies on the following functional $J_{8}$ instead of $J_{6}$ (defined in (3.100)):

$$
J_{8}(t):=\int_{0}^{L} \int_{0}^{+\infty} g(s) \int_{t-s}^{t} \psi_{x}^{2}(x, \tau) d \tau d s d x .
$$

The functional $J_{8}$ is well-defined and satisfies, for all $\left.\lambda \in\right] 0,1[$,

$$
J_{8}^{\prime}(t) \leq-(1-\lambda) \xi(t) J_{8}(t)-\lambda \int_{0}^{L} \int_{0}^{+\infty} g(s) \psi_{x}^{2}(x, t-s) d s d x+g_{0} \int_{0}^{L} \psi_{x}^{2}(x, t) d x,
$$

which is similar to (3.101) but without the last term of (3.101). See Ref. 20 in case (1.5) for more details concerning the idea of proof.

6. If the second initial data $\psi_{1}$ satisfies

$$
\int_{0}^{L} \int_{0}^{+\infty} \psi_{1}^{2}(x, s) d s d x<+\infty
$$

the condition (3.5) is not needed, and the estimates (3.9) and (3.12) hold for $\gamma: \mathbb{R}_{+} \rightarrow \mathbb{R}_{+}^{*}$ of class $C\left(\mathbb{R}_{+}, \mathbb{R}_{+}^{*}\right)$ and nonincreasing such that

$$
|f(t-s)| \geq \gamma(t) \int_{t}^{+\infty}|f(\tau-s)| d \tau, \quad \forall t \in \mathbb{R}_{+}, \quad \forall s \in[0, t] .
$$

Replacing (3.5) by (5.5) allows $f$ to have more general decay rate at infinity; so the growth of $f$ does not depend on the one of $g$. The idea of proof (see Ref. 20 for (1.5)) relies on the following functional $J_{9}$ instead of $I_{7}$ (defined in (3.52)):

$$
J_{9}(t):=2 \int_{0}^{L} \int_{0}^{+\infty}|f(s)| \int_{t-s}^{t} \psi_{t}^{2}(x, \tau) d \tau d s d x .
$$

As for (5.3) with $\lambda=\frac{1}{2}$, we find

$$
J_{9}^{\prime}(t) \leq 2 \alpha g_{0} \int_{0}^{L} \psi_{t}^{2}(x, t) d x-\gamma(t) J_{9}(t)-\int_{0}^{L} \int_{0}^{+\infty}|f(s)| \psi_{t}^{2}(x, t-s) d s d x,
$$

which is similar to (3.54) with $2 \alpha g_{0}$ and $J_{9}$ instead of $g(0) \beta_{0}$ and $I_{7}$, respectively. Similarly, if both (5.1) and (5.4) are satisfied, then (3.9) holds under (5.5) instead of (3.5).

7. As in the distributed delay case, if the first initial data $\psi_{0 x}$ satisfies (5.1), then (3.9) holds for the discrete time delay case (4.4), where $\phi(t)=\int_{0}^{t} \min \{1, \xi(s)\} d s$. The idea of proof consists in replacing $J_{6}$ (defined in (3.100)) by $J_{8}$ (defined in (5.2)).

\section{ACKNOWLEDGMENTS}

The author is grateful for the continuous support and the kind facilities provided by KFUPM. This work has been funded by KFUPM under Project No. IN121013. The author would like to 
express his gratitude to the anonymous referees for helpful and fruitful comments, and very careful reading.

${ }^{1}$ F. Alabau-Boussouira, P. Cannarsa, and V. Komornik, "Indirect internal stabilization of weakly coupled evolution equations," J. Evol. Eq. 2, 127-150 (2002).

${ }^{2}$ F. Ammar-Khodja, A. Benabdallah, J. E. Muñoz Rivera, and R. Racke, "Energy decay for Timoshenko systems of memory type," J. Differential Equations 194, 82-115 (2003).

${ }^{3}$ A. Benaissa, A. K. Benaissa, and S. A. Messaoudi, "Global existence and energy decay of solutions for the wave equation with a time varying delay term in the weakly nonlinear internal feedbacks," J. Math. Phys. 53, 123514 (2012).

${ }^{4}$ M. M. Cavalcanti, V. N. Domingos Cavalcanti, F. A. Falcão Nascimento, I. Lasiecka, and J. H. Rodrigues, "Uniform decay rates for the energy of Timoshenko system with the arbitrary speeds of propagation and localized nonlinear damping," Z. Angew. Math. Phys. (published online).

${ }^{5}$ C. M. Dafermos, “Asymptotic stability in viscoelasticity,” Arch. Rational Mech. Anal. 37, 297-308 (1970).

${ }^{6}$ R. Datko, J. Lagnese, and M. P. Polis, "An example on the effect of time delays in boundary feedback stabilization of wave equations," SIAM J. Control Optim. 24, 152-156 (1986).

${ }^{7}$ R. Datko, "Two questions concerning the boundary control of certain elastic systems," J. Differential Equations 92, 27-44 (1991).

${ }^{8}$ H. D. Fernández Sare and J. E. Muñoz Rivera, "Stability of Timoshenko systems with past history,” J. Math. Anal. Appl. 339, 482-502 (2008).

${ }^{9}$ H. D. Fernández Sare and R. Racke, "On the stability of damped Timoshenko systems: Cattaneo versus Fourier's law," Arch. Rational Mech. Anal. 194(1), 221-251 (2009).

${ }^{10}$ A. E. Green and P. M. Naghdi, "A re-examination of the basic postulates of thermomechanics," Proc. R. Soc. London A 432, 171-194 (1991).

${ }^{11}$ A. E. Green and P. M. Naghdi, "On undamped heat waves in an elastic solid,” J. Thermal Stress. 15, 253-264 (1992).

${ }^{12}$ A. E. Green and P. M. Naghdi, "Thermoelasticity without energy dissipation," J. Elasticity 31, 189-208 (1993).

${ }^{13}$ A. Guesmia, "Quelques résultats de stabilisation indirecte des systèmes couplés non dissipatifs," Bull. Belg. Math. Soc. 15, 479-497 (2008).

${ }^{14}$ A. Guesmia, “Asymptotic stability of abstract dissipative systems with infinite memory," J. Math. Anal. Appl. 382, 748-760 (2011).

${ }^{15}$ A. Guesmia, "Well-posedness and exponential stability of an abstract evolution equation with infinite memory and time delay," IMA J. Math Control Inform. 30, 507-526 (2013).

${ }^{16}$ A. Guesmia and S. A. Messaoudi, "On the control of solutions of a viscoelastic equation," Appl. Math. Comput. 206, 589-597 (2008).

${ }^{17}$ A. Guesmia and S. A. Messaoudi, "General energy decay estimates of Timoshenko systems with frictional versus viscoelastic damping," Math. Meth. Appl. Sci. 32, 2102-2122 (2009).

${ }^{18}$ A. Guesmia and S. A. Messaoudi, "On the stabilization of Timoshenko systems with memory and different speeds of wave propagation,” Appl. Math. Comput. 219, 9424-9437 (2013).

${ }^{19}$ A. Guesmia, S. A. Messaoudi, and A. Soufyane, "On the stabilization for a linear Timoshenko system with infinite history and applications to the coupled Timoshenko-heat systems," Elec. J. Differential Equations 2012, 1-45 (2012).

${ }^{20}$ A. Guesmia and N. Tatar, "Some well-posedness and stability results for abstract hyperbolic equations with infinite memory and distributed time delay," J. Differential Equations (submitted).

${ }^{21}$ M. Kirane and B. Said-Houari, "Existence and asymptotic stability of a viscoelastic wave equation with a delay," Z. Angew. Math. Phys. 62, 1065-1082 (2011).

${ }^{22}$ V. Komornik, Exact Controllability and Stabilization: The Multiplier Method (John Wiley, Paris, 1994).

${ }^{23}$ J. E. Lagnese, Boundary Stabilization of Thin Plates (SIAM, Philadelphia, 1989).

24 J. E. Lagnese and J. L. Lions, Modelling, Analysis and Control of Thin Plates (Collection RMA, Masson, Paris, 1988).

${ }^{25}$ S. A. Messaoudi and M. I. Mustafa, "A stability result in a memory-type Timoshenko system," Dynam. Syst. Appl. 18, 457-468 (2009).

${ }^{26}$ S. A. Messaoudi and B. Said-Houari, "Uniform decay in a Timoshenko-type with past history," J. Math. Anal. Appl. 360, 459-475 (2009).

${ }^{27}$ M. I. Mustafa, "Exponential decay in thermoelastic systems with boundary delay," J. Abst. Differ. Equ. Appl. 2, 1-13 (2011).

${ }^{28} \mathrm{~S}$. Nicaise and C. Pignotti, "Stability and instability results of the wave equation with a delay term in the boundary or internal feedbacks," SIAM J. Control Optim. 45, 1561-1585 (2006).

${ }^{29}$ S. Nicaise and C. Pignotti, "Stabilization of the wave equation with boundary or internal distributed delay," Diff. Integral Equ. 9-10, 935-958 (2008).

${ }^{30} \mathrm{~S}$. Nicaise and C. Pignotti, "Interior feedback stabilization of wave equations with time dependent delay," Elect. J. Differential Equations 41, 1-20 (2011).

${ }^{31}$ S. Nicaise, C. Pignotti, and J. Valein, "Exponential stability of the wave equation with boundary time-varying delay," Disc. Cont. Dyn. Syst. Ser. S 3, 693-722 (2011).

${ }^{32}$ S. Nicaise, J. Valein, and E. Fridman, "Stability of the heat and of the wave equations with boundary time-varying delays," Disc. Cont. Dyna Syst. Ser. S 2, 559-581 (2009).

${ }^{33}$ V. Pata, "Exponential stability in linear viscoelasticity," Quart. Appl. Math. 3, 499-513 (2006).

${ }^{34}$ A. Pazy, Semigroups of Linear Operators and Applications to Partial Differential Equations (Springer-Verlag, New York, 1983).

${ }^{35}$ B. Said-Houari, "A stability result for a Timoshenko system with past history and a delay term in the internal feedback," Dyn. Syst. Appl. 20, 327-354 (2011). 
${ }^{36}$ N. E. Tatar, "Exponential decay for a viscoelastic problem with a singular kernel," Z. Angew. Math. Phys. 60, 640-650 (2009).

${ }^{37}$ N. E. Tatar, "On a large class of kernels yielding exponential stability in viscoelasticity," Appl. Math. Comput. 215, 2298-2306 (2009)

${ }^{38}$ N. E. Tatar, "How far can relaxation functions be increasing in viscoelastic problems?," Appl. Math. Lett. 22, 336-340 (2009).

${ }^{39}$ N. E. Tatar, "A new class of kernels leading to an arbitrary decay in viscoelasticity," Mediterr. J. Math. 6, 139-150 (2010).

${ }^{40}$ N. E. Tatar, "On a perturbed kernel in viscoelasticity," Appl. Math. Lett. 24, 766-770 (2011).

${ }^{41}$ N. E. Tatar, "Arbitrary decays in linear viscoelasticity,” J. Math. Phys. 52, 013502 (2011).

${ }^{42}$ N. E. Tatar, "Uniform decay in viscoelasticity for kernels with small non-decreasingness zones," Appl. Math. Comput. 218, 7939-7946 (2012).

${ }^{43}$ N. E. Tatar, "Oscillating kernels and arbitrary decays in viscoelasticity," Math. Nachr. 285, 1130-1143 (2012).

${ }^{44}$ S. Timoshenko, "On the correction for shear of the differential equation for transverse vibrations of prismatic bars," Philos. Mag. 41, 744-746 (1921). 